C. G. Galizia $\cdot$ B. Kimmerle

\title{
Physiological and morphological characterization of honeybee olfactory neurons combining electrophysiology, calcium imaging and confocal microscopy
}

Received: 19 April 2003/ Revised: 20 October 2003 / Accepted: 22 October 2003/Published online: 25 November 2003

(C) Springer-Verlag 2003

\begin{abstract}
The insect antennal lobe is the first brain structure to process olfactory information. Like the vertebrate olfactory bulb the antennal lobe is substructured in olfactory glomeruli. In insects, glomeruli can be morphologically identified, and have characteristic olfactory response profiles. Local neurons interconnect glomeruli, and output (projection) neurons project to higher-order brain centres. The relationship between their elaborate morphology and their physiology is not understood. We recorded electrophysiologically from antennal lobe neurons, and iontophoretically injected a calciumsensitive dye. We then measured their spatio-temporal calcium responses to a variety of odours. Finally, we confocally reconstructed the neurons, and identified the innervated glomeruli. An increase or decrease in spiking frequency corresponded to an intracellular calcium increase or decrease in the cell. While intracellular recordings generally lasted between 10 and $30 \mathrm{~min}$, calcium imaging was stable for up to $2 \mathrm{~h}$, allowing a more detailed physiological analysis. The responses indicate that heterogeneous local neurons get input in the glomerulus in which they branch most strongly. In many cases, the physiological response properties of the cells corresponded to the known response profile of the innervated glomerulus. In other words, the large variety of response profiles generally found when comparing antennal lobe neurons is reduced to a more predictable response profile when the innervated glomerulus is known.
\end{abstract}

C. G. Galizia · B. Kimmerle

Institut für Biologie-Neurobiologie, Freie Universität Berlin, Königin Luise Str. 28-30, 14195 Berlin, Germany

C. G. Galizia $(\bowtie)$

Entomology 383, University of California,

Riverside, CA 92521, USA

E-mail: galizia@ucr.edu

Fax: + 1-909-7873086

B. Kimmerle

Olympus BioSystems, Robert-Koch-Str 9, 82152 Planegg, Germany
Keywords Antennal lobe Calcium response · Honeybee $\cdot$ Olfactory sensory neuron · Olfactory glomerulus

Abbreviations $A C T$ antenno-cerebralis-tract $\cdot A L$ antennal lobe $\cdot A P$ action potential $\cdot l-A C T$ lateral $\mathrm{ACT} \cdot L N$ local neuron $\cdot L P L$ lateral protocerebral lobe $\cdot m$ - $A C T$ medial ACT $\cdot M B$ mushroom body $O S N$ olfactory sensory neuron $\cdot P N$ projection neuron $\cdot T 1$ tract 1 of the antennal nerve

\section{Introduction}

Information about odours in the environment is detected by olfactory sensory neurons (OSNs). In insects, their axons project to the antennal lobe (AL), the first olfactory centre in the brain, which shows striking similarity both in function and architecture to the mammalian olfactory bulb (Hildebrand and Shepherd 1997). Both the AL and the olfactory bulb have a glomerular organization. A variety of studies employing a multitude of techniques have shown that these olfactory glomeruli act as functional units in the olfactory code (Boeckh and Tolbert 1993; Hildebrand and Shepherd 1997; Hansson and Christensen 1999; Xu et al. 2000; Galizia and Menzel 2001). Each glomerulus gets strongly convergent input from one (or maybe a few) sensory neuron families expressing the same receptor, thus acting as a sort of collection basket of similar response profiles. LNs interconnect olfactory glomeruli, and the processed, or 'reformatted' (Laurent 1999; Friedrich and Laurent 2001) activity is relayed to higher order brain centres by the projection neurons (PNs). The PNs are equivalent to the mammalian mitral/tufted cells, while the local neurons (LNs) correspond to the mammalian periglomerular and granule cells. Substantial information is available about the cellular properties of neurons selective for sexual pheromones, especially in moths, be it at the level of OSNs, LNs, PNs or their 
innervated glomeruli (Hansson et al. 1992; Christensen et al. 1993; Berg et al. 1998; Vickers et al. 1998; Hansson and Christensen 1999).

For non-pheromonal odours, knowledge about the chemical response profiles of OSN populations is increasing (de Bruyne et al. 1999, 2001; Hansson and Anton 2000; Røstelien et al. 2000; Stranden et al. 2002). Response properties of PNs to non-pheromonal odours have also been published, for moths (Kanzaki et al. 1989; King et al. 2000; Sadek et al. 2002) and for locusts (Laurent et al. 1996). In the latter species Laurent and co-workers investigated the importance of spike timing for olfactory coding (MacLeod and Laurent 1996; Wehr and Laurent 1996; Laurent et al. 2001). In honeybees, PN responses to non-pheromonal odours have also been described (Homberg 1984; Flanagan and Mercer 1989b; Sun et al. 1993; Stopfer et al. 1997; Abel et al. 2001; Müller et al. 2002).

Much less is known about the response profiles of LNs. While these are non-spiking neurons in locusts (MacLeod and Laurent 1996), they do spike in honeybees (Flanagan and Mercer 1989b; Sun et al. 1993). In honeybees, there may be as many as 4,000 LNs (Witthöft 1967). There are two morphological types of LNs, the homogeneous (homo-LNs) and the heterogeneous (hetero-LNs) interneurons (Flanagan and Mercer 1989b; Sun et al. 1993). Homo-LNs branch in a large number of glomeruli over wide areas of the AL, innervating each glomerulus in a similar fashion with only few profiles. Hetero-LNs strongly innervate a single glomerulus, and diffusely branch into several (between 20 and over 100) other glomeruli (Flanagan and Mercer 1989b; Fonta et al. 1993). Both LN types differentially respond to different odours: they have excitatory responses to some odours and inhibitory responses (i.e. a reduction in the background spiking activity) to others (Sun et al. 1993). A population of about 800 LNs expresses GABAergic immunoreactivity (Schäfer and Bicker 1986), while a smaller population of about 35 LNs stain with an antihistamine antibody (Bornhauser and Meyer 1997). Hetero-LNs are more frequently labelled with intracellular electrodes (Flanagan and Mercer 1989b; Fonta et al. 1993), suggesting that there may be more heteroLNs than homo-LNs. Nevertheless, the relationship between morphological type and transmitter remains as yet unknown.

Most PNs are uniglomerular, i.e. they only branch in one glomerulus. Generally, they do not have any collaterals in any other part of the $\mathrm{AL}$, apart from short protrusions at the AL exit (Müller et al. 2002). PNs leave the AL either via the lateral (1-ACT) or the medial antenno-cerebralis tract $(\mathrm{m}-\mathrm{ACT})$. Both tracts innervate the mushroom body (MB) calyces, and the lateral protocerebral lobe (LPL). When stimulated with an odour, PNs respond by increasing or reducing their spike rates with respect to background activity, or with complex sequences of both excitation and inhibition (Flanagan and Mercer 1989a; Sun et al. 1993; Abel et al. 2001; Müller et al. 2002). In addition, there is a small population of multiglomerular and GABAergic PNs which leave the AL via the medio-lateral antennocerebralis tract (ml-ACT) and end in the LPL (Schäfer and Bicker 1986; Flanagan and Mercer 1989b; Sun et al. 1993).

The cell bodies of AL neurons (both PNs and LNs) are located around the AL neuropil in several clusters, the most prominent of which are the medial and the lateral cell clusters. Both clusters contain somata of both PNs and LNs.

The olfactory glomerulus is the morphological and functional unit of the AL. The arrangement and number of glomeruli is characteristic for each species, and individual glomeruli are recognizable across individuals of one species (Rospars and Chambille 1981; Rospars 1988). This has permitted the creation of morphological atlases, which can be used to identify glomeruli and thus to compare physiological results at a glomerular level across individuals. Such atlases are now available for several species, such as the honeybee, with about 160 glomeruli (Flanagan and Mercer 1989a; Galizia et al. 1999a) and the fruit fly with about 43 glomeruli (Stocker et al. 1990; Laissue et al. 1999). The nomenclature used for glomerular identity differs between species. In honeybees, glomeruli are identified by the name of the antennal tract innervating them (T1-T4) and a number (e.g. T1-33 or T3-45). In Drosophila, a very clear convergence of OSNs expressing the same receptor gene to one or two glomeruli has been shown (Vosshall et al. 2000). In honeybees, calcium imaging studies have shown that each glomerulus has a characteristic olfactory response profile (Galizia and Menzel 2001). This has allowed us to start creating a 'functional atlas' of glomerular physiology (Galizia et al. 1999b; Sachse et al. 1999).

From these studies it appears likely that there is a strong relationship between the physiological response properties of a neuron that branches strongly in a particular glomerulus, be it an OSN, a LN or a PN, and the overall response profile of that glomerulus. However, while we know that almost all neuron types in the AL make synapses onto each of the other neuron types (with the exception that while PNs synapse onto LNs, no report to date has shown them to synapse onto an OSN) (Gascuel and Masson 1991; Malun 1991; Distler and Boeckh 1998), little is known about the polarity of LNs, about the spatial distribution of activity within a neuron, and about the olfactory response profiles of individual neurons with respect to the identity of the innervated glomeruli. Addressing these questions, we electrophysiologically recorded olfactory responses from AL neurons using sharp electrodes. We have then iontophoretically injected a neuronal tracer and a calcium sensitive dye into the neuron, and measured the spatiotemporal calcium dynamics of the same neurons while exposing the animal to the same odours as before. Finally we confocally reconstructed the morphology of the stained neurons, and morphologically identified the innervated glomeruli. 


\section{Materials and methods}

Overall experimental design and sequence of events

Forager honeybees (Apis mellifera carnica) were caught at the hive entrance. Beehives were kept in an indoor flight-room during the winter. Bees were gently chilled, and mounted in a Plexiglas stage. A window was cut into the cuticle, and glands and tracheae were removed to expose the ALs. At all times, the brain was kept wet with artificial saline solution (in $\mathrm{mmol}^{-1}: \mathrm{NaCl} \mathrm{130;} \mathrm{KCl} 6$; $\mathrm{MgCl}_{2}$ 5; sucrose 160; glucose 25; HEPES 10; pH 6.7; 500 mosmol) at room temperature $\left(22^{\circ} \mathrm{C}\right)$. After successful electrophysiological recordings, the preparation was covered with a cover slip, and moved to another setup for optical imaging. Therefore, electrophysiological recordings and calcium measurements were never simultaneous. Finally, the brain was fixed in formaldehyde $(4 \%)$, removed from the head capsule, dehydrated, cleared in methyl salicylate, embedded in Permount, and confocally analysed.

The odours tested were 2-heptanone (HPN), isoamyl acetate (ISO, which is present in plant extracts and also a component of the bee sting-pheromone), citral (CIT) and geraniol (GER) (the latter two are components of the Nasanov-gland pheromone) and a series of aliphatic alcohols: 1-hexanol (HX1), 1-heptanol (HP1), 1-octanol (OC1), 1-nonanol (NO1) (all from Sigma, Deisenhofen). The aliphatic hydrocarbons were diluted in mineral oil and adjusted for vapour pressure (for a total of $1,000 \mu \mathrm{l}$, we used $20 \mu \mathrm{l}$ hexanol, $70 \mu \mathrm{l}$ heptanol, $165 \mu \mathrm{l}$ octanol or $250 \mu \mathrm{l}$ nonanol, respectively). Furthermore, extracts from clove oil (CLV) and orange blossoms (ORG) were tested. Not all odours were tested in all animals. Odours $(4 \mu \mathrm{l})$ were placed on filter paper $\left(1 \mathrm{~cm}^{2}\right)$ into $1-\mathrm{ml}$ plastic cartridges, which were inserted into a computer-controlled olfactometer. This olfactometer switches between a continuous airstream and the odour-laden stimulus, thus minimising mechanical stimulus artefacts (Galizia et al. 1997). Control stimuli were pure air (i.e. clean filter paper) and mineral oil. The concentration of the odour stimuli used was high but not saturating: the same odour concentrations are routinely used in behavioural studies in the institute, and honeybee show no difficulty in learning these stimuli in an appetitive associative paradigm and easily differentiate between different stimuli at this concentration.

\section{Electrophysiological recordings}

Borosilicate glass electrodes were pulled with a horizontal puller (P97; Sutter Instruments). Electrodes were slowly advanced into the AL using a micromanipulator (Narishige). The tips of the electrodes were filled with a cocktail of the calcium-indicator Fura-2 (Molecular Probes, Eugene) and Alexa-568 (Molecular Probes, Eugene). The long-wavelength neuronal tracer Alexa was used because of its stability for morphological analysis in the confocal microscope, and because of its fluorescence at a wavelength where honeybee brains have limited background $(568 \mathrm{~nm})$. More precisely: $1 \mathrm{mg}$ Fura was diluted in $20 \mu \mathrm{KOH}\left(5 \mathrm{mmol} \mathrm{l}^{-1}\right)$, while $1 \mathrm{mg}$ Alexa was diluted in $22.8 \mu \mathrm{KCl}\left(100 \mathrm{mmol} \mathrm{l}^{-1}\right)$. These two stock solutions were frozen in aliquots. The tip solution was prepared by mixing $2 \mu \mathrm{l}$ of each stock solution with $2 \mu \mathrm{l}$ HEPES $\left(100 \mathrm{mmol}^{-1}, \mathrm{pH} 7.3\right)$. Electrodes were backfilled with $3 \mathrm{~mol} \mathrm{l}^{-1}$ $\mathrm{KCl}$. Resistance in the tissue was generally between 150 and $250 \mathrm{M} \Omega$ (mean 204, range 100-350). In control experiments, calcium green was used instead of Fura-2. No qualitative differences were seen between the results from the two dyes, but the signal-tonoise ratio was better when optically recording with Fura. In some preparations Alexa was not used; in these instances, no confocal analysis was possible after the physiological characterization. Successful penetration of a cell was recorded as a jump in the baseline, and generally accompanied with background spiking activity, or a burst of spikes due to the initial penetration into the cell. Recordings were done in bridge mode using an intracellular amplifier (BRAMP 1, NPI Electronics). Data were digitized on a 1401 interface and stored on disk using Spike2 2.3 software
(Cambridge Electronic Design, UK). The olfactometer was also controlled from the Spike2 software. After testing responses to odours, a hyperpolarizing current $(1-5 \mathrm{nA})$ was applied for 3-10 min in order to iontophoretically fill the cell with dye. Odours were tested also during hyperpolarisation in order to ensure that the electrode was still within the target cell. Once cell contact was lost, the preparation was removed and transferred to the imaging setup. Data analysis of the electrophysiological recordings was performed using Spike2. Spike sorting was performed using the template-matching algorithm implemented in Spike2.

\section{Calcium imaging}

Optical recordings were carried out using an Axioscope microscope (Zeiss, Germany), a 20× LD NA $=0.6$ air objective (Leica, Germany), and a calcium imaging system (Till-Photonics, Germany). Images were taken while switching excitation between $340 \mathrm{~nm}$ and $380 \mathrm{~nm}$ at a rate of six double images per second $(6 \mathrm{~Hz})$. In control experiments $5 \mathrm{~Hz}$ and $10 \mathrm{~Hz}$ were also tested, with no qualitative differences. It should be noted that the temporal resolution of the calcium measurements is much lower than the electrophysiological recordings. Exposure times varied across preparations, with typical values of $80-100 \mathrm{~ms}$ for $340 \mathrm{~nm}$ and $10-20 \mathrm{~ms}$ for $380 \mathrm{~nm}$. Dichroic mirror was $410 \mathrm{~nm}$, emission filter $440 \mathrm{LP}$. In control experiments using calcium green, the excitation light was $488 \mathrm{~nm}$, dichroic 500 , emission filter $510 \mathrm{LP}$, exposure time around $100 \mathrm{~ms}$, and recording frequency $5 \mathrm{~Hz}$. The CCD was used with a binning-on-chip value of 2 in both axes, resulting in a pixel size of $2.4 \times 2.4 \mu \mathrm{m}$ at the preparation. The true optical resolution was less, being limited by light scattering and inappropriate corrections of the air objective for a living aqueous specimen. Data acquisition and olfactometer control were done with Till-Photonics software.

Data analysis of imaging data was done using software customwritten in IDL (RSI, Boulder, Colo., USA). Raw data were filtered with a median filter to remove salt-and-pepper noise. Bleaching over time was corrected by subtracting a logarithmic curve fitted to the brightness profile of the entire frame. This procedure subtracts the same value from each pixel within a frame, but a different value for different frames, hence correcting for bleaching without affecting the spatial activity patterns. Then, the ratio of each $340 \mathrm{~nm}$ image and the corresponding $380 \mathrm{~nm}$ was calculated. For time-courses, an area of $7 \times 7$ pixels, corresponding to $15 \times 15 \mu \mathrm{m}$ was taken. False-colour-coded images represent the values at maximum response after stimulus, minus the values immediately preceding stimulation. Unless indicated, all data shown are single-trial measurements

\section{Confocal analysis}

Brains were mounted in Permount between two cover slips spaced $1 \mathrm{~mm}$ apart. A Leica TCS 4D confocal with a DM RBE microscope was used. The excitation laser line was $568 \mathrm{~nm}$, emission was LP590. Stacks of confocal images were taken across the entire AL using a $20 \times$ LD Leica NA $=0.6$ objective. In some instances, details were recorded at higher magnification. Confocal data analysis, neuropil segmentation and neuron reconstruction were performed with Amira software (Indeed, Germany; http://www.amiravis.com). In preparations with more than one stained neurons (e.g. Fig. 6) we only included the neuron with the strongest staining for clarity. Weakly stained neurons could be followed along their strongest processes only, which were excluded from the reconstruction.

\section{Identification of glomeruli}

In many preparations the most strongly innervated glomerulus could be identified based on the confocal stacks. In these cases, the background fluorescence was sufficient to see the borders of the 
neighbouring olfactory glomeruli. The glomeruli were identified by comparing the data stack with the digital atlas of the AL (Galizia et al. 1999a; http://www.neurobiologie.fu-berlin.de/honeybeeALatlas). In some instances, glomerular borders were visible in the CCD images, and glomeruli could be identified from these images using the same method. In all cases, glomerular identification was done solely on the basis of morphological characteristics, such as shape and relative position.

\section{Results}

This paper is based on a total of 47 successful intracellular electrophysiological recordings. Of these, 28 were successfully loaded with a calcium-sensitive dye (either calcium green or Fura-2), and their odour responses optically recorded. Ten recordings were from PNs (see below), the remaining 18 recordings were from LNs. In 24 cases the staining was suitable for successive confocal imaging. All LNs were heterogeneous, i.e. they strongly innervated a single glomerulus, and diffusely innervated many other glomeruli. Most recordings from LNs were multiple-cell recordings, with multiple spike amplitudes in the electrophysiological traces, and several cell bodies stained with the dye. In five cases two individual cells could be recognized, in two cases three cells could be seen, resulting in a total of 27 LNs. In a total of 14 cases (either PN or LN) the innervated glomerulus could be unambiguously identified based on its morphological characteristics, in eight further cases identification was only approximate. In the remaining cases no identification was possible.

\section{Projection neurons}

Of ten recorded PNs, seven were identified as PNs based on the confocal images in which an axon leaving the AL was visible. Three additional neurons had insufficient staining for the confocal analysis, but were also classified as PNs on the basis of their response properties (spikes of uniform height and shape, see below). All PNs recorded were uniglomerular PNs with axons leaving the $\mathrm{AL}$ via the 1ACT tract towards the lateral protocerebrum and the mushroom bodies. All neurons innervated glomeruli of the region innervated by the antennal nerve tract $\mathrm{T} 1$, corresponding to the area into which we inserted the electrodes. Six neurons showed excitatory odour responses (i.e. stimulus-correlated increases in action potential frequency, or increased intracellular calcium concentration, Fig. 1), and two also showed inhibitory responses (Fig. 3). In all cases intracellular calcium response profiles matched the electrophysiological observation, i.e. odours that elicited an increase in action potentials also showed an increase in intracellular calcium concentration, and a decrease in action potentials corresponded to a calcium concentration decrease. In two cases the calcium response was broader: in one case a neuron with no spontaneous activity in the electrophysiological recording showed spontaneous
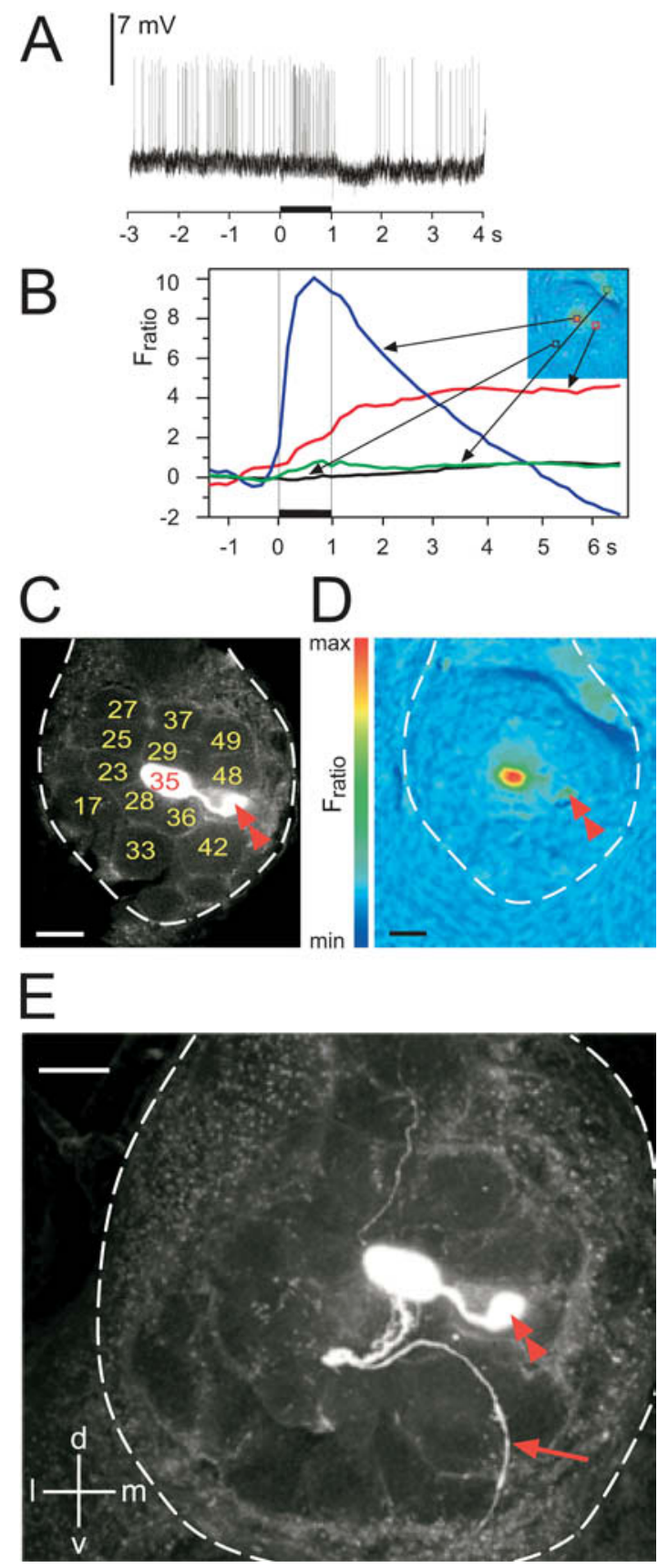

activity consisting of many bursts in the calcium traces, and in another case a neuron which showed no odour responses at all in the electrophysiological recording showed weak responses to hexanol in the calcium imaging. Table 1 gives the response properties of the neurons with the innervated glomerulus.

Neurons that responded to an odour with increased spiking (Fig. 1A) also responded with an increase in intracellular calcium concentration (Fig. 1B). In the CCD image, the neuron showed clear Fura fluorescence (Fig. 1D), and both the soma, the glomerulus and the connecting primary neurite were visible in the confocal image (Fig. 1C). The soma was located in the medial cell cluster. Since only one neuron was filled with Fura, the 
Fig. 1A-E Uniglomerular projection neuron (PN). Spike frequency increase corresponds to increased calcium. A Electrophysiological response to clove oil. Upon stimulation (black bar) a burst of action potentials occurred. After stimulation the neuron was inhibited, with no action potentials and a decrease in membrane potential. Note the high spontaneous activity and the spikes of uniform height. B Calcium response to clove oil in the glomerular dendrites (dark blue), the soma (red), and reference areas within the antennal lobe (AL) of the cell shown in A. Stimulus time is given by the bar and vertical lines. The intracellular calcium concentration in the glomerulus rapidly increases upon stimulation. Since the increase is already strong in the first frame, and measurement points are interconnected, the curve appears to rise before stimulus onset. Calcium concentration decreases after stimulation, and falls below baseline. Calcium in the soma also increases, but with a delay and more slowly, and does not decrease during the sampled period. No activity is apparent in the remainder of the AL (green and black lines). $\mathbf{C}$ View of the same specimen with the CCD camera. In this AL, the glomerular borders were clearly visible, allowing the identification of glomerulus 35 of the antennal nerve tract 1 (T1-35) as the innervated glomerulus. Note the large soma (red double arrowhead) and the strong labelling in the glomerulus, due to dense dendritic branching. The approximate AL borders are indicated with dashed lines (also in $\mathbf{D}$ and $\mathbf{E}$ ). Scale bar $50 \mu \mathrm{m}$. D False-colour coded image of the calcium response to clove oil (compare to B). Note the strong signal in the glomerulus, the weaker signal in the soma, and a very weak signal in the primary neurite connecting soma and glomerular dendrites. Also note the absence of signals in the remainder of the AL. E Confocal extended focus view of this neuron (compare with $\mathbf{C}$ ). Note the clear staining of the axon leaving the AL towards the protocerebrum (red arrow). Scale bar $50 \mu \mathrm{m}$. Compare with Fig. 2 remainder of the $\mathrm{AL}$ gave no calcium responses (see trace in Fig. 1B). Upon odour stimulation, the intracellular calcium increased both in the glomerulus, with a rapid and strong increase, and in the soma with a much slower increase. No axon was visible at the resolution of the CCD images. Odour offset led to a transient cessation of background activity in the electrophysiological trace. The calcium concentration in the glomerulus dropped immediately at stimulus offset, going below baseline after $5 \mathrm{~s}$. In contrast, calcium concentration in the soma kept increasing for several seconds after stimulus offset. The borderlines of the glomeruli were visible in both the CCD image (Fig. 1C, D), and in an extended focus view of the confocal image stack (Fig. 1E). This allowed identification of the innervated glomerulus, which in this preparation was T1-35. The confocal analysis showed that a single PN was strongly labelled. In this particular preparation, a single axon of a receptor neuron was also labelled (faintly visible in Fig. 1E, leaving dorsally). From the confocal sections, we reconstructed the entire morphology of this neuron (Fig. 2). The reconstructed neuron is shown in three projection views. Note the superficial position of the soma (Fig. 2B), and the very dense innervation of the glomerulus (Fig. 2D). Also, the axon leaves the glomerulus in a contorted fashion, descending the entire AL with a loop, before exiting via the 1ACT towards the MBs. Besides the dense branching in the AL, the axon

Table 1 Response properties of projection neurons (PNs) and local neurons (LNs) innervating identified glomeruli

\begin{tabular}{|c|c|c|c|c|c|}
\hline $\mathrm{PN} / \mathrm{LN}$ & $\begin{array}{l}\text { Innervated } \\
\text { glomerulus }\end{array}$ & $\begin{array}{l}\text { Exitatory } \\
\text { response to: }\end{array}$ & $\begin{array}{l}\text { Inhibitory } \\
\text { response to: }\end{array}$ & $\begin{array}{l}\text { Spontaneous } \\
\text { activity }\end{array}$ & Atlas \\
\hline $\mathrm{LN}$ & $\mathrm{T} 1-11 / 22 ?$ & $\mathrm{OC} 1$ & None & Regular & No atlas data \\
\hline LN & T1-12? & CLV & None & None & Insuff. atlas data \\
\hline LN & T1-15? & ORG & None & None & Insuff. atlas data \\
\hline $\mathrm{PN}$ & $\mathrm{T} 1-21$ & None & None & Irregular & No data overlap \\
\hline $\mathrm{PN}$ & T1-29 (Fig. 3) & Citral & Orange & Regular & Exit. to citral not expected \\
\hline LN & T1-29 (Fig. 7) & $\mathrm{HPN}$ & ISO, OC1, HP1 & Regular & Exit. to ISO expected-no match! \\
\hline LN & $\mathrm{T} 1-30$ & None & None & None & Match \\
\hline $\mathrm{PN}$ & T1-33 (Fig. 8) & $\mathrm{OC} 1, \mathrm{NO}^{\mathrm{a}}$ & None & Bursty & Match \\
\hline $\mathrm{PN}$ & T1-33 (Fig. 8) & $\mathrm{OCl}$ & Weak to ISO & Regular & Match \\
\hline LN & T1-33? (Fig. 8) & OC1, weak to HX1 & None & Bursts & Match \\
\hline $\mathrm{PN}$ & T1-35 (Figs. 1, 2) & Clove oil & After clove oil & Irregular & Exit. to clove oil not expected \\
\hline $\mathrm{PN}$ & $\mathrm{T} 1-36$ & ISO, HP1, HPN & None & Regular & Match \\
\hline LN & T1-36 (Fig. 7) & $\begin{array}{l}\text { OC1, ISO, HP1, } \\
\text { HPN }\end{array}$ & None & None & Match \\
\hline $\mathrm{PN}$ & $\mathrm{T} 1-38$ & Orange & None & Irregular & Exit. to ISO expected but not found \\
\hline LN & T1-38 (Fig. 7) & HP1 & None & None & Exit. to ISO expected-no match! \\
\hline $\mathrm{PN}$ & T1-46 (?) (Fig. 4) & None $e^{\mathrm{b}}$ & None & Bursts & No data overlap \\
\hline LN & $\mathrm{T} 1-51 ?$ & NO1, HPN & None & Irregular & No data \\
\hline LN & $\mathrm{T} 1-52$ & $\mathrm{HX} 1, \mathrm{OC} 1$ & None & Regular & (Weak responses to $\mathrm{HX} 1$, OC1) \\
\hline $\mathrm{PN}$ & $\mathrm{T} 1-52(?)$ & None & None & Bursts & (Weak responses to HX1 expected) \\
\hline $\mathrm{LN}$ & $\mathrm{T} 1-55$ & HX1, weak to OC1 & None & None & No data \\
\hline LN & $\mathrm{T} 1-60 ?$ & ORG, CLV & None & None & CLV: match, org: no match \\
\hline LN & $\mathrm{T} 3-31$ & ORG, CLV & None & None & No data \\
\hline $\mathrm{PN}$ & (?) Medial & None & None & Bursts (imaging only) & \\
\hline
\end{tabular}

Electrophysiological and calcium responses during odour stimulation corresponded. Glomerular identification is based on morphological position. Where the glomerular identification is uncertain, a question mark is added. The column to the right (Atlas) indicates how the observed responses relate to the published physiological atlas of AL glomeruli (Galizia et al. 1999b; Sachse et al. 1999) a Neuron responded to HX1 in the calcium imaging, but not during the electrophysiological measurement

${ }^{\mathrm{b}}$ Neuron responded once to citral. This response was not reproducible

$C L V$ clove oil; HP1 1-heptanol; HPN 2-heptanon; $H X 1$ 1-hexanol; ISO isoamyl acetate; NOI 1-nonanol; none no response to the tested odours; OC1 1-octanol; ORG orange extract 


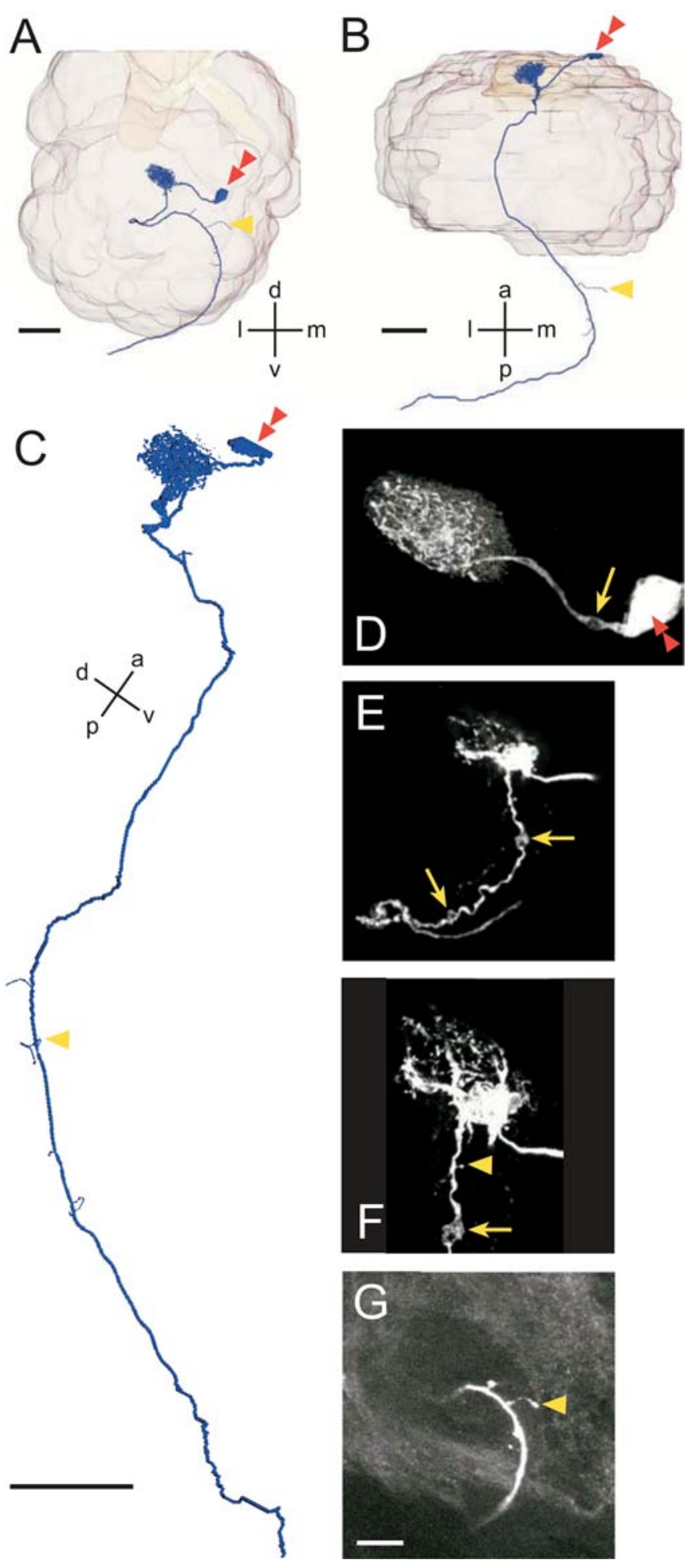

gave rise to several short fibres very close to the glomerulus (Fig. 2F), and again at the exit point of the AL (Fig. 2G). A few varicosities were visible both along the axon and in the neurite connecting the glomerulus and the soma (Fig. 2D, E, F). These may be sites of synaptic interactions, but their precise nature is unclear.

PNs often showed sustained spontaneous background activity (e.g. Figs. 1 and 3). When odour
Fig. 2A-G Three-dimensional reconstructions of the preparation in Fig. 1. The neuron is shown in blue, with the main glomerulus as a filled structure, while the approximate shape of the AL is indicated in faint colour. Reconstruction by U. Schröter. A Frontal view; B coronal view; $\mathbf{C}$ parasagittal view, rotated. Scale bar in $\mathbf{A}-$ C: $50 \mu \mathrm{m}$. D-G Details of the neuron from the confocal sections, extended focus views. D Soma (double arrowhead) and glomerulus with the connecting neurite. The neurite has swellings (arrows). Note the very fine arborisation in the glomerulus, which is sectioned peripherally. E The glomerulus at a more central level, with the neurite to the soma (right) and the axon (down). Note the intricate path the axon takes, and the swellings along the axon (arrows). F Close-up view of the glomerular output region. Note how the branching at this internal focal depth is different within the glomerulus as compared to D. There are several spine-like structures along the axons. G Close up view of the axon as it exits the AL. At this location there are several protrusions, one of them quite long (arrowhead). Scale bar $50 \mu \mathrm{m}$

stimulation led to an inhibition, with hyperpolarizing membrane potential and a cessation of action potential firing (Fig. 3A), the same odour also led to a decrease in intracellular calcium concentration (Fig. 3B, C). Interestingly, in the particular case shown in Fig. 3, the calcium concentration had a strong rebound calcium increase after stimulus offset (Fig. 3B-D), which was not paralleled by a similar increase in spiking frequency in the electrophysiological recording (Fig. 3A). This neuron had a poor signal-to-noise ratio in the calcium imaging, and the morphological staining was too weak for confocal imaging. Nevertheless, the innervated glomerulus could be identified as T1-29 in the CCD images (Fig. $3 \mathrm{E}-\mathrm{G})$.

In most cases neurons that showed spontaneous activity, which consisted of bursts in the electrophysiological record, also had irregular changes in calcium concentration in optical measurements. Figure 4 shows an example of a uniglomerular PN with strong and burst-like spontaneous activity. This neuron did not respond to any of the tested odours, with the exception of a single response to citral (Fig. 4A). This response was not reproducible, and started before stimulus. The response may have been facilitated by its temporal coincidence with a spontaneous activity burst. In the electrophysiological trace, this neuron displayed bursts of spontaneous activity intermitted by stretches of no or only limited activity (Fig. 4B). The intracellular calcium concentration was also very irregular. Nevertheless, the one response to citral led to much stronger calcium increase than any of the spontaneous bursts (Fig. 4C). The glomerulus innervated by this neuron could not be identified.

Neurons local to the AL

We recorded a total of 27 LNs. All LNs were heterogeneous LNs, i.e. they innervated a single glomerulus strongly, and many others diffusely. In 8 cases the innervated glomerulus could be identified, in 5 more 

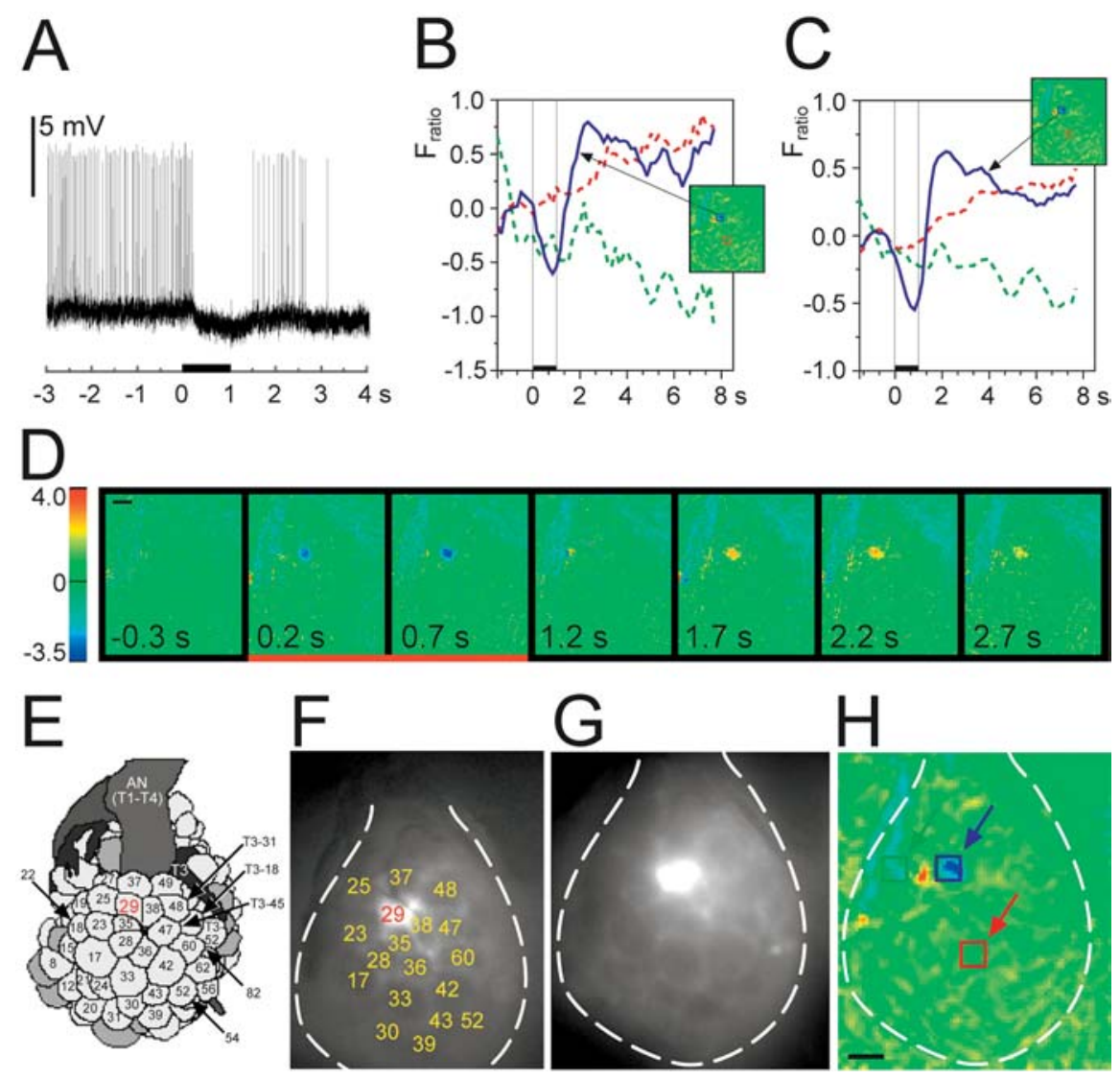

Fig. 3A-H Uniglomerular PN. Spike frequency decrease corresponds to reduced calcium. A Electrophysiological response to orange oil. Stimulation leads to a complete cessation in background spiking activity, and a drop in membrane potential. After stimulation, activity recovers. Note the uniform spike height and the high background activity. Over long recording stretches background activity was not as regular as before this stimulus. This is also apparent in the drop of activity after the rebound spike train, which was not consistent across trials. B Time-courses for the responses to orange oil in glomerulus T1-29 (dark blue line) and two reference areas. Evaluated positions are indicated in $\mathbf{H}$. Note the decrease in calcium concentration during stimulation and the increase thereafter (dark blue line). C Average of 8 stimulations with orange. Except for the reduced noise the curves correspond to the single trial measurement in B. D Sequence of false-colour coded activities when stimulating with orange (single trial). Every $3 \mathrm{rd}$ frame is shown, i.e. two frames per second. The red bar gives stimulus timing. Intracellular calcium concentration decreases in glomerulus T1-29 upon stimulation, and increases thereafter. The activity is highly focalised in one spot, with no activity in the rest of the AL. Scale bar $50 \mu \mathrm{m}$. E Schematic view of the standard atlas of the honeybee AL, with the glomerular identities. F Identification of the glomeruli in this specimen based on their morphology. Glomerular borders have been contrast-enhanced in order to make them easily recognisable. A larger field-of-view is shown in this image than in $\mathbf{D}, \mathbf{G}$ and $\mathbf{H}$. $\mathbf{G}$ Fluorescent view of the stained neuron and the AL with the CCD camera. The glomerular borders are visible, and glomerulus T1-29 is brightly fluorescent. H Falsecolour coded response to orange oil, which elicits a decrease in calcium concentration. The red area close to the glomerulus is a movement artefact (compare with $\mathbf{D}$ ). Evaluation areas for $\mathbf{B}, \mathbf{C}$ are marked with squares. The approximate AL borders are indicated with dashed lines in $\mathbf{F}-\mathbf{H}$. Scale bar $50 \mu \mathrm{m}$ cases the identification is approximate. The glomeruli innervated by the remainder could not be identified. Fifteen cells had the same olfactory-response profile in the electrophysiological and the imaging recordings; in two cases there were responses to more odours in the calcium records than in the electrophysiology. In one additional case the response-spectrum of the two methods matched, but the calcium responses were much longer. In 3 cells the electrophysiological and optophysiological response-spectra were similar except for some odours. Three out of 27 cells had response-spectra that were inconsistent between the electrophysiological and imaging experiments. Neurons differed widely in their basic electrophysiological properties, with some being spontaneously and tonically active, while others only fired when stimulated with odours. Both inhibitory and excitatory responses were seen: 24 neurons showed excitatory responses, 6 neurons also showed inhibitory responses, 3 neurons showed no odour responses at all.

Electrophysiological recordings generally had multiple spike heights (Figs. 5, 7). Spikes of different heights had different odour response profiles. In Fig. 5A, orange-extract led to increased activity of the large spikes. On the other hand, the response to clove oil consisted mainly of small spikes. In the calcium imaging of this preparation, both orange and clove oil led to strong, focalized calcium increases, but the two activity spots were separate, i.e. they corresponded to two different glomeruli, which were direct neighbours. The calcium 


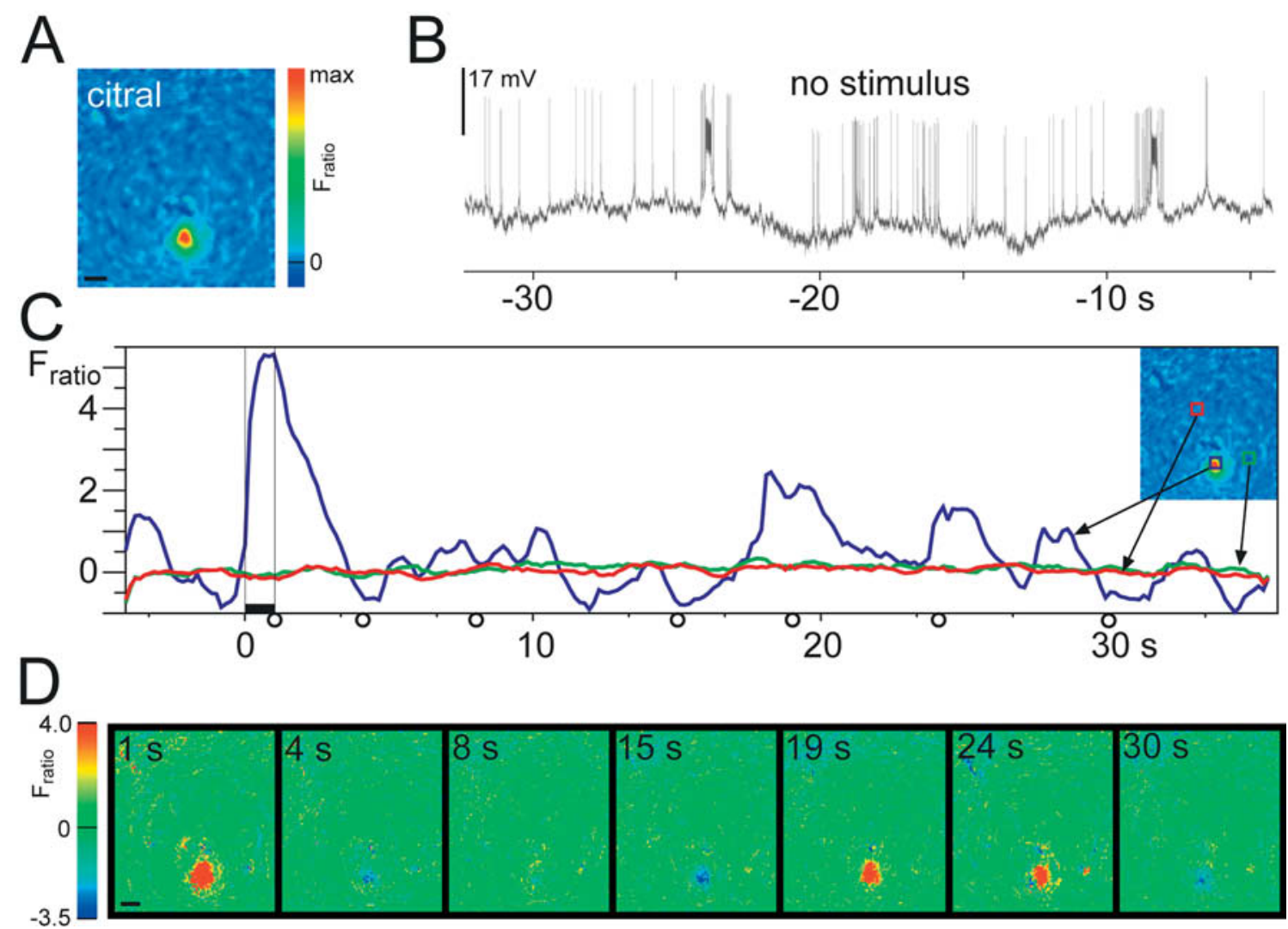

Fig. 4A-D Spontaneous activity match for electrical and calcium activity in uniglomerular PNs. A False-colour image of calcium response to stimulation with citral. The only active area corresponds to the innervated glomerulus. No activity is visible in the remainder of the AL. Scale bar $50 \mu \mathrm{m}$. B Electrophysiological recording of spontaneous activity, consisting of single spikes and bursting instances. Note the uniform height of action potentials and the fluctuating resting potential. No stimulus was given. C Stretch of $40 \mathrm{~s}$ calcium recording from the same preparation, with a citral stimulus given (stimulus time is indicated by bar and vertical lines). Note the strongly fluctuating intracellular calcium concentration. The calcium response to citral started before stimulation, but was much higher than any of the spontaneous events. Other trials with citral stimulation, and citral stimulation during electrophysiological recording, gave no responses. Circles along the time axis indicate positions of frames shown in $\mathbf{D}$. The time-axis is identical to B. D False-colour coded images from the same measurement. The numbers in each frame indicate seconds from stimulus onset. The first frame (1 s) is within the response to citral. Note the decrease in calcium concentration at $15 \mathrm{~s}$ and $30 \mathrm{~s}$, and the increase at $19 \mathrm{~s}$ and $24 \mathrm{~s}$ (compare to the trace in C). Scale bar $50 \mu \mathrm{m}$

increase was very strong in the responding glomerulus (Fig. 5C), with a weak response in other areas of the AL as well. Interestingly, orange, which elicited only few (large) spikes in the electrophysiological record, led to a stronger increase in intracellular calcium than clove oil, which elicited many (but small) spikes. The time-course was similar to the glomerular response found in PNs: a strong increase during stimulus, with a drop in the intracellular calcium after stimulus offset. However, no 'undershoot' was observed, i.e. calcium concentration returned to baseline, but not below. The confocal analysis of the preparation showed that several neurons were stained. In this preparation, three distinct somata were visible. The neurites extended from the somata into the AL, and branched diffusely into wide areas of the AL. The dense innervation of one glomerulus is clearly visible in Fig. 5D, panel iii; the main glomeruli of the other two neurons could not be identified. This unequal staining of the neurons was a common feature in recordings from multiple LNs. The visible glomerulus was deep within the AL. We therefore exclude the possibility that this was one of the two glomeruli whose activity was recorded with calcium imaging. Imaging with the CCD camera only gives access to the superficial layer of the AL. Consequently, the two superficial glomeruli that gave very strong calcium responses were not strongly stained in the confocal afterstaining. The diffuse innervation in the remainder of the AL showed interesting features: some glomeruli were diffusely innervated, while club-like, fairly gross processes innervated other glomeruli. This morphological heterogeneity suggests that these LNs may have different physiological roles in different sub-branches. Alternatively, the three neurons had differing properties. In the morphological reconstruction of this preparation (Fig. 6A) only the clearly visible processes were included. While most of the AL was innervated, some areas were not, and others differed strongly in the degree to which they were innervated. We estimate that most of the reconstruction in Fig. 6A derives from a single neuron, which would again confirm the idea that the computational role that this neuron plays differs in different areas of the AL. 

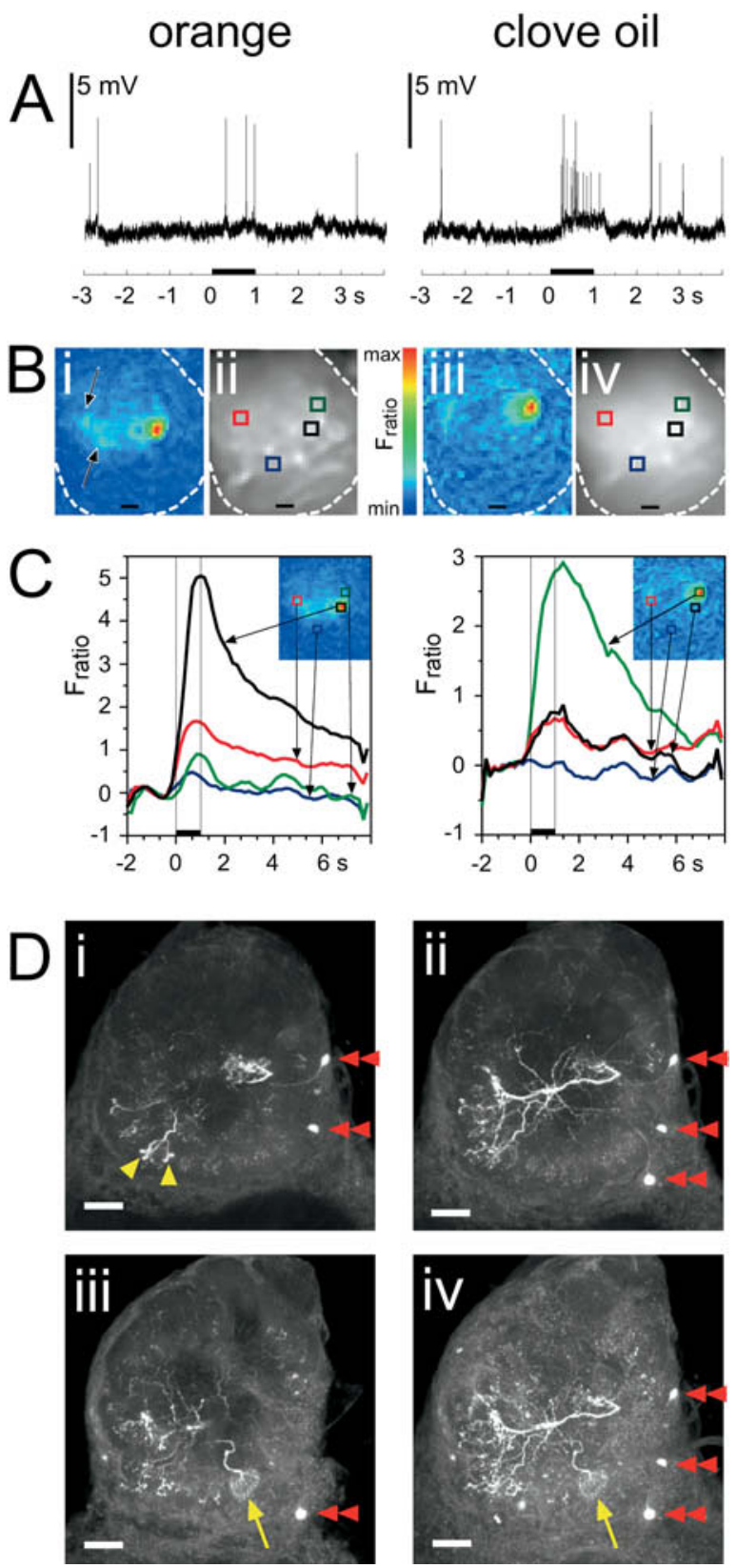

LNs, just like PNs, could respond to odour stimulation with either intracellular calcium increase or decrease. Figure 6B and Fig. 7 show a preparation in which three neurons were stained, as seen by the three somata visualized in the confocal preparation (Fig. 7F). The electrophysiological record showed a sustained background activity, and responses to several odours. However, spikes had different sizes and shapes. Using a spike-sorting algorithm we distinguished at least three spike types. With this sorting, the responses to the three odours 1-heptanol, 1-octanol and isoamyl acetate were clearly distinct (Fig. 7B). In the subsequent calciumimaging recordings, four activity foci were visible, while no activity was found in the remainder of the $\mathrm{AL}$ (Fig. 7C). The time-courses of calcium activity are shown in Fig. 7D. From the morphological view in
Fig. 5A-D Recording from a group of two heterogeneous local neurons (LNs). A Electrophysiological responses to orange and clove oil. The bar gives stimulus timing. Two distinct response heights were apparent in this recording, both with only little background activity. Stimulating with orange elicited a response of large action potentials (APs), but no small APs. Clove oil elicited a strong burst of small APs. B Calcium imaging of this preparation. The false-colour coded images (colour scale at the centre) have been scaled to the maximum response in each image. The approximate AL borders are indicated with dashed lines. Scale bar $50 \mu \mathrm{m}$. Panel $i$ : stimulation with orange led to a strongly focalised response, associated with weak activity in diffuse areas of the AL (black arrows). Panel ii: the morphological image in the CCD camera (black and white contrast enhanced image) does not reveal any clear structure. Panel iii: stimulation with clove oil elicits a strongly focalized response in an area distinct and adjacent to that activated by orange. Panel iv: non-contrast enhanced morphological image with the areas evaluated in $\mathbf{C}$ superimposed onto it. C Time-traces of calcium responses in different areas of the $\mathrm{AL}$ as response to orange (left) and clove oil (right). Note that the maximum calcium response to clove oil is weaker than that to orange and that the two odours activate clearly different areas. D Extended focus views from the confocal stacks of this preparation. Scale bar $50 \mu \mathrm{m}$. Panel $i$ : upper slices. Panel ii: intermediate slices. Panel iii: bottom slices. Panel iv: all slices from the frontal view. Note the three cell bodies to the right (red double arrowheads) and the strongly labelled glomerulus in the bottom slices (yellow arrow in panel iii). The position of this glomerulus does not correspond to any of the two responsive glomeruli shown in B. Also note the dense network of cellular processes all across the AL, with different shapes such as the diffuse branching patterns seen in most glomeruli, and the club-shaped innervation in some glomeruli (yellow arrowheads in panel $i$ )

Fig. 7E, it appears that one active spot corresponded to a soma, while the other three were glomeruli. One glomerulus had activity that was consistently preceding the activity seen in the soma, both for calcium increases as well as for decreases (full and dotted green lines in Fig. 7D), suggesting that they belong to the same neuron. The response in the soma was always weaker than in the glomerulus. Comparing the electrophysiological response and the calcium responses showed that for each of the three glomerular calcium-response profiles, there was a corresponding response profile in the electrophysiology: one cell was inhibited by 1-heptanol and 1-octanol, but did not respond to isoamyl acetate; one cell responded to 1-heptanol only; and one responded to all three odours, with the strongest response to 1-octanol. The morphological analysis showed that the three cells were not equally stained. Indeed, one of the three active glomeruli was not visible in the morphological view, and the second neuron was only weakly stained. The third neuron, however, was very bright in the confocal image, and has been reconstructed (Fig. 6B). Its morphology corresponded to a heterogeneous LN, with a densely innervated glomerulus, and a diffusely innervated remainder of the AL. This neuron did not display the club-shaped processes seen in Fig. 6A, and innervated a more restricted area of the AL. From comparing the morphological reconstruction with the calcium images, it is apparent that only the main glomerulus responded to odour stimulation with changes in intracellular calcium. 

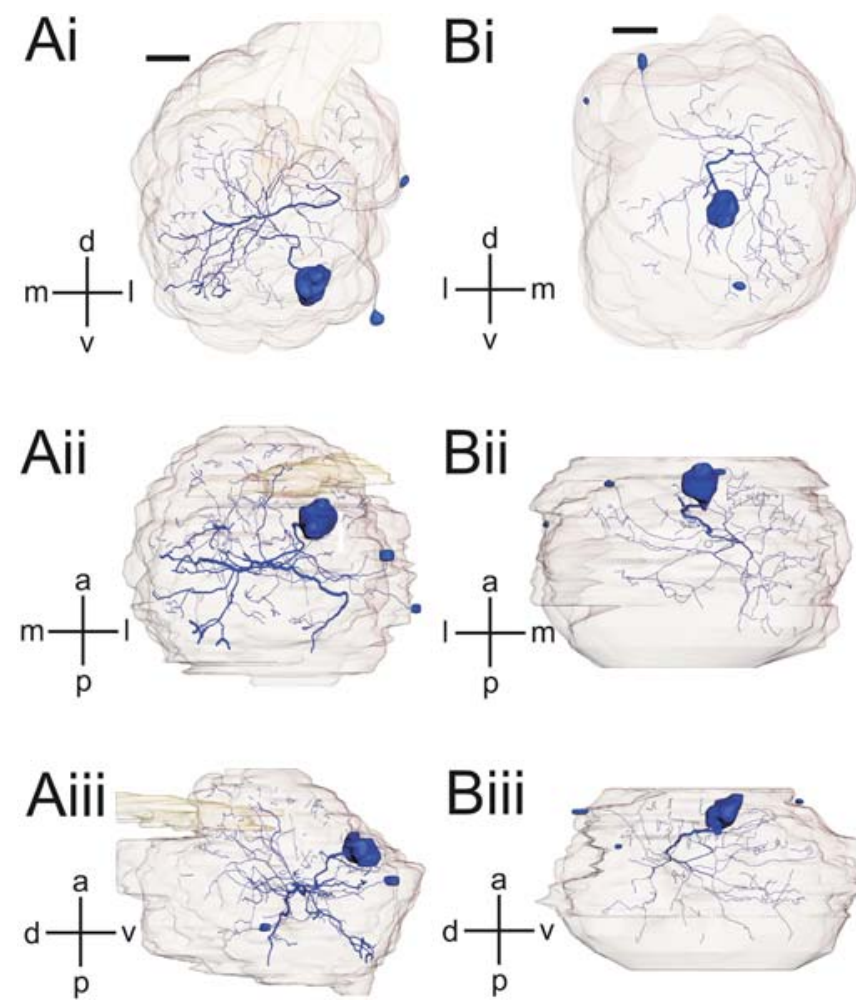

Fig. 6A,B Three-dimensional reconstructions of two preparations in which heterogeneous LNs were stained. $i$ : frontal view; ii: coronal view; iii: parasagittal view. A same preparation as in Fig. 5. B same preparation as in Fig. 7. Scale bars $50 \mu \mathrm{m}$. In both cases only one neuron could be reliably traced due to unequal staining. Branches unequivocally belonging to the other stained neurons have not been included. The very fine arborisation at the terminal ends of the neurites could not be included in the segmentation due to lack of contrast in the images. The neurons are shown in blue, with the main glomerulus as a filled structure, while the approximate shape of the $\mathrm{AL}$ is indicated in faint colour. Reconstruction by U. Schröter. Note how large areas of the AL are diffusely innervated in both cases. Also note how the diameters of the neurite differ: the neurons have a central tree with very large diameter, and fine arborisation with very thin diameter

\section{Match of neuron responses with AL atlas}

The glomeruli of the honeybee AL are stereotype, and can be recognized among animals based on their morphology. Furthermore, their odour response spectra appear to be genetically defined and have been characterized for some glomeruli and odours using imaging of bath-applied calcium-sensitive dyes (Galizia et al. 1999b; Sachse et al. 1999). All the PNs in our sample were uniglomerular PNs. All LNs were heterogeneous ones. For both classes, the main innervation site is an individual glomerulus that can, in principle, be identified. We therefore asked whether the odour-responses that we found corresponded to the responses found in the physiological atlas. In order to make such a comparison, the innervated glomerulus must be morphologically identifiable, and be in an area that has been functionally characterized.
Fig. 7A-F Recording from a group of three heterogeneous LNs. A Electrophysiological responses to 1-heptanol, 1-octanol, isoamyl acetate. The bar gives stimulus timing. Note the high background activity and the spikes of different height. B Spike sorting based on template matching reveals distinct groups of spikes, which have been extracted and colour-coded in this image. For clarity, spikes in different groups have been shifted vertically. Background activity is dominated by the 'green' spike, the response to 1-heptanol by the 'blue' and 'red' spike with an inhibition in the 'green' spike, the response to 1-octanol is dominated by the 'red' spikes, again with a drop in the 'green', and isoamyl acetate leads to an increase in 'red' spikes with a minor drop in the 'green' spike form. C False-colour coded calcium responses in the AL to the same odours. Changes in activity are limited to four areas that correspond to three glomeruli (arrows) and one soma (green double arrowhead). 1-Heptanol leads to calcium decrease in one glomerulus (green arrow) and an increase in the other two (blue and red arrows), 1-octanol to a strong decrease in one (green arrow and the soma) and an increase in another (red arrow), with no change in the third glomerulus (blue arrow), while the effect of isoamyl acetate is limited to calcium increase in one glomerulus (red arrow). The approximate $\mathrm{AL}$ borders are indicated with dashed lines. Scale bar $50 \mu \mathrm{m}$. D Time traces of calcium in different areas of the AL. Note how the soma (dashed green) trails its corresponding glomerulus (continuous green) in the calcium response. E Identification of the glomeruli in this preparation. In this image, glomerular boundaries are visible. Note the strong fluorescence in glomerulus T1-36 and the weak fluorescence in glomerulus T1-38. The prefix T1 is omitted from all T1 glomeruli. F Confocal extended focus view of the stained neurons. Three somata are clearly stained, two of which (to the right, white double arrows) were not in the field-of-view in the calcium-imaging measurements, while the third one corresponds to the soma in the calcium imaging (green double arrow). Note the densely stained glomerulus (red arrow), the weakly stained glomerulus (blue arrow) and the diffuse innervation of the remainder of the AL. Scale bar $50 \mu \mathrm{m}$ in $\mathbf{E}$ and $\mathbf{F}$

The innervated glomerulus could be identified in nine PN preparations (in two only tentatively, see Table 1). In three cases the recorded odour responses corresponded to the expectations; in two cases there was no data overlap, i.e. the odours tested in this study are not present in the physiological atlas for that glomerulus. In two cases the neurons responded to an odour that was not expected from the physiological atlas: the neuron in glomerulus T1-29 responded to citral and the neuron in glomerulus T1-35 responded to clove oil, though, according to the atlas, both responses were not expected. In two cases the neurons did not show an expected response: the neuron in glomerulus T1-38 would have been expected to respond to isoamyl acetate, but did not respond to that odour. A weak response to hexanol would have been expected in the neuron innervating T152 , but that neuron did not respond to any odour.

The situation for LNs is more complex. Here, several preparations consisted of multiple recordings, with more than one cell responding, and consequently more than one main glomerulus being labelled. Nevertheless, out of seven cells with clear glomerular attribution, only three showed a mismatch between atlas and observed responses. However, even though the morphological identification of the stained neuron is clear in these instances, the correspondence of the morphologically labelled glomerulus and the imaged glomerulus may be 

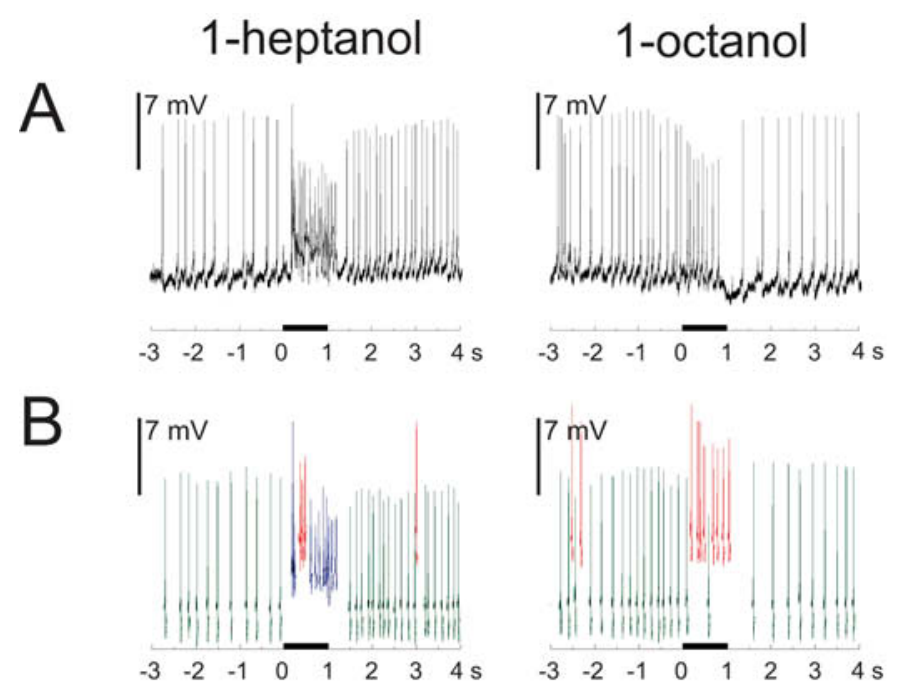

isoamylac.
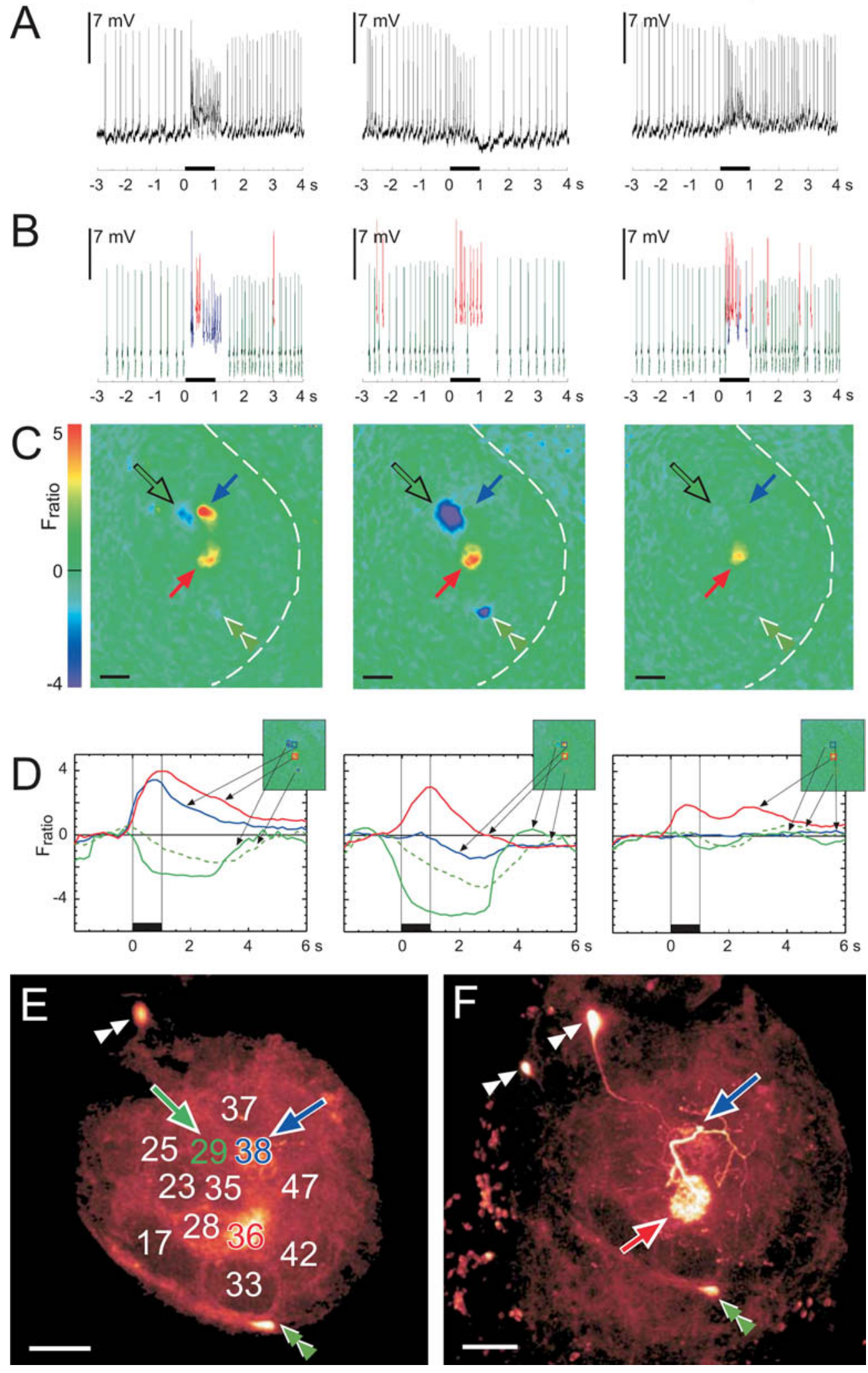

ambiguous. Indeed, some glomeruli that give very clear responses can fail to appear in the confocal reconstructions, even though their soma is labelled (e.g. glomerulus T1-29 in Fig. 7).

For glomerulus T1-33 we found three cells: two PNs, with unambiguous glomerular identity, and one heterogeneous LN, with less clear identification. All three neurons responded strongly to octanol in the electro- 


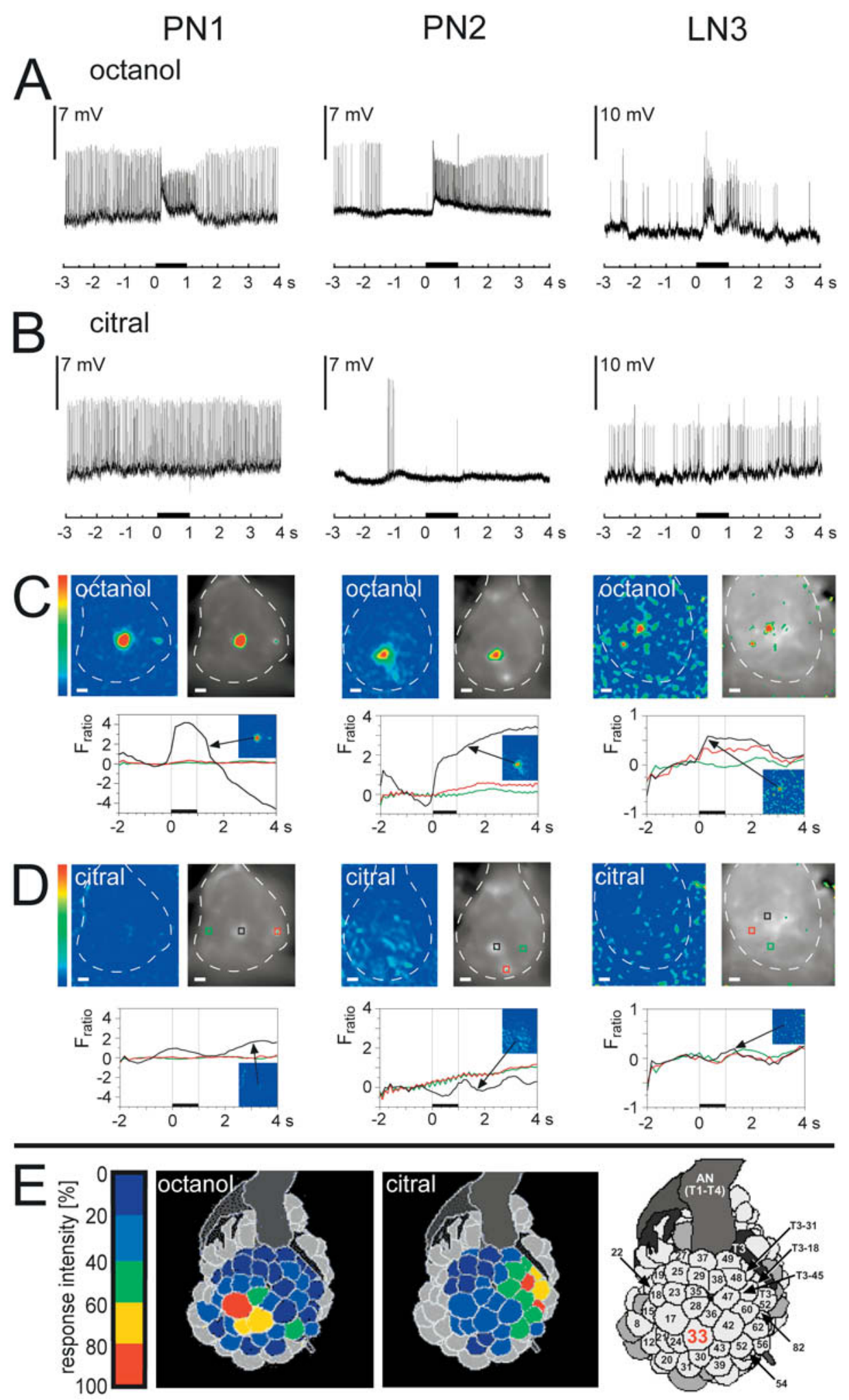

physiological recording (Fig. 8A) but not to citral (Fig. 8B). Similarly, calcium imaging showed strong responses to octanol (Fig. 8C) and no responses to citral (Fig. 8D). These responses correspond to the physiological atlas, which shows that glomerulus T1-33 is strong in the response to octanol but not to citral
(Fig. 8E). Despite their matching odour responses, the two PNs differed in other properties: one neuron had a strong spontaneous activity, both in the electrophysiological and in the calcium recording. This neuron responded to octanol with a strong burst and calcium increase, but stopped responding at stimulus offset, with 
Fig. 8A-E Responses to odours in three different cells each innervating glomerulus T1-33. The figure is arranged in three columns, representing three different neurons. Left and middle columns: two PNs (PN1 and PN2, glomerulus identification reliable); right column: $L N$ (glomerulus identification tentative). A Electrophysiological response to octanol in the three neurons. Each neuron responded to the stimulus with strong excitation. $P N 1$ : a strong burst exceeding the tonic spontaneous activity. $P N 2$ : a long-lasting train of APs. Note the two different spike heights in the LN. B Electrophysiological responses to citral. None of the three neurons responded to citral. The two lines at stimulus onset and offset in the central neuron are the stimulus artefacts from the valve-controlled odour delivery system. C Calcium responses to octanol for the three neurons. For each neuron the false-colour coded response (upper left) and the threshold response overlaid to the morphological image are shown. False-colour sequence is shown on the left. Each neuron is scaled to its own maximum response. Time traces for three selected areas are given: glomerulus 33 (black), the soma (red) and a reference area (green). The identification of the soma location for the $\mathrm{LN}$ is tentative. Note the prolonged calcium response in PN2 that matches its prolonged response in $\mathbf{A}$. The signals from the LN to the right were very weak, resulting in a bad signal-to-noise ratio. D Calcium responses to citral for the three neurons. Note the total lack of response in all three neurons. Also note the spontaneous changes in calcium concentration in the two PNs, matching their spontaneous electrophysiological responses. The positions taken for the timetraces are the same as in $\mathbf{C}$ and are indicated by squares in the contrast-enhanced morphological view. The approximate AL borders are indicated with dashed lines in $\mathbf{C}$ and $\mathbf{D}$. Scale bar $50 \mu \mathrm{m}$ in $\mathbf{C}$ and D. E Glomerular activity patterns elicited by octanol and citral from the honeybee functional AL atlas (http:// www.neurobiologie.fu-berlin.de/honeybeeALatlas), and schematic view of the AL for identification of individual glomeruli. Glomerulus T1-33 is excited by octanol but not by citral

calcium concentration going below baseline. The other neuron had less baseline activity, but the calcium responses were strong and lasted long after stimulus offset. In fact, maximum calcium concentration was reached at a considerable delay with respect to the stimulus. The response in the $\mathrm{LN}$ was weak. In all three neurons, the cell body (or putative cell body in the case of the LN) also responded to octanol, but much less strongly.

\section{Discussion}

In this study we analysed the spatio-temporal properties of AL neurons in the honeybee, using a combination of electrophysiological, optophysiological and confocal techniques. We found that increase or decrease in spiking frequency corresponded to intracellular calcium increase or decrease in the cell. While intracellular recordings of these neurons could generally be kept for between 10 and $30 \mathrm{~min}$, calcium imaging of the labelled neuron was stable for up to $2 \mathrm{~h}$. This allowed a more detailed characterization of their physiological properties. In many cases the physiological response properties of the cells corresponded to the response profile of the innervated glomerulus as described in the physiological AL atlas (Galizia et al. 1999b; Sachse et al. 1999). In other words, the great variety of response profiles generally found when comparing AL neurons reduces to a more predictable response profile when the innervated glomerulus is known.

\section{Methodological aspects}

This study combines three methods: electrophysiology with iontophoretic dye injection, calcium imaging and confocal microscopy. The three methods were applied in sequence, and are therefore separated in time. However, we directly compared results from the three methods. How reliable are the conclusions? We would like to discuss sequentially some possible error sources.

\section{Labelling specificity}

Some dye could be released from the electrode before the target cell has been impaled, and be taken up by other cells. In this case, the optical methods would detect information from more cells than the electrophysiology. The same consequences could be expected if the dye were to exit the target cell, either when the electrode is withdrawn, or when the membrane or the impalement site is permeable. Because the dyes used are membrane impermeable, re-uptake should be negligible. But even if re-uptake were to occur, it seems unlikely that a particular, individual cell would be the only one to sequestrate the spilled dye. In both cases, therefore, we would expect diffuse staining as a result of the dye spillage. In the confocal images, this staining would create some background, reducing the signal-to-noise ratio, but not further affect the images. In the calcium imaging, such a staining should result in a situation reminiscent of a bath-applied dye. We would then expect to also get responses to odours in those glomeruli which are not most strongly innervated by the stained cells, and in particular we would expect responses in those glomeruli which are known to give the strongest responses to a particular odour, for example in glomeruli T1-17 and T1-33 when stimulating with 1-nonanol (Sachse et al. 1999). This was never the case, so that we can exclude this possible artefact.

There is, however, a labelling specificity issue that directly affected our results when the recorded neuron was a hetero-LN. In these cases, we generally recorded from more than one neuron simultaneously. The individual neurons could be distinguished in the electrophysiological records by their spike size and shape. In the calcium imaging, different glomeruli gave best responses to different odours, and each glomerulus corresponded in its response profile to one spike shape in the electrophysiological record. However, the amount of Fura visible in the three cells was unequal, based on the resting fluorescence. Similarly, the fluorescent staining with Alexa in the confocal images differed among neurons. Strikingly, the amount of Fura in the neurons did not match the amount of Alexa visible in the confocal images, indicating that the two dyes differ in how they are transported within and between cells. In the example 
given in Fig. 7 three glomeruli showed clear calcium responses. Indeed, in the confocal images, three somata were visible, but only two innervated glomeruli, and these two were unequally stained. In the confocal images, the amount of dye directly results in visible signal. In calcium measurements using Fura, however, the dye concentration does not directly affect the size of the signals, since taking the ratio of the two excitation wavelengths corrects for staining intensity. Therefore, weakly stained cells can also give strong signals. Nevertheless, unequal staining may result in some cells that could be invisible in both the calcium imaging and in the confocal reconstructions, even though they were recorded electrophysiologically.

\section{Intracellular multiunit recordings}

In the LN recordings we generally recorded from more than one neuron, resulting in electrophysiological recordings with multiple spike heights and forms. This phenomenon has been reported both for honeybees (Flanagan and Mercer 1989b) and for moths (Christensen et al. 1993). Why did we and others get multiple units for LNs, but not for PNs? We impaled the LNs within or close to the coarse neuropil. In this area, many LNs form extensive branching arbours, with closely apposed processes (Gascuel and Masson 1991; Fonta et al. 1993). The electrode may have formed an artificial and very local syncytium between the neurons, which would explain the multiple unit recordings. This happens only rarely in PNs, because they do not branch in the coarse neuropil, but rather extend a very fine axon out of the AL (compare Fig. 2 with Fig. 6). Alternatively, the multiple units seen in the electrophysiological recordings of LNs may reflect single LNs with multiple spike initiation zones (Flanagan and Mercer 1989b). However, the data presented in this paper argue against this view: in all cases where we had multiple units in the electrophysiological recordings, we also saw multiple glomeruli responding in the calcium-imaging recordings and multiple somata stained. These strongly responsive glomeruli corresponded to the main glomeruli of heteroLNs. Since hetero-LNs do not have more than one main glomerulus (Fonta et al. 1993), the recordings were from multiple neurons rather than from one neuron with multiple functional subunits. Finally, multiple spike heights may also arise from neurons interconnected by gap-junctions. However, in all cases that we measured, the multiple units were neurons with little or no functional overlap in their response spectra. This is not what would be expected for electrically coupled cells, thus corroborating our view that different spike shapes derive from distinct LNs. Nevertheless, this does not exclude the possibility that these cells may be functionally coupled in a more complex fashion.

When we had multiple unit recordings, we used spikesorting algorithms to attribute individual spikes to the units. The algorithm sorts spikes on the basis of their shape into different categories. This method can give false results, in particular when a neuron responds to one odour with a strong train of APs, a situation where the individual spikes are often reduced in size and thus display a different shape from before the burst. In this study, however, we took advantage of the dual approach combining electrophysiology and calcium imaging. Since we could match the different spike categories to different glomeruli and thus to different cells in the calcium measurements (Figs. 5, 7) we are confident that the spike-sorting algorithm was accurate in separating individual cells.

\section{Glomerular identification}

How reliably can glomeruli be recognized based on their morphology? A series of studies has shown that the number, layout and position of glomeruli is stereotypic in insects (Rospars 1988), allowing for the creation of glomerular atlases in several species (Flanagan and Mercer 1989a; Galizia et al. 1999a; Laissue et al. 1999; Berg et al. 2002). Nevertheless, identifying a glomerulus innervated by a single cell is not necessarily straightforward. In concordance with others facing the same identification task (Laissue et al. 1999), we found that a combination of strategies are necessary. Some glomeruli are clearly identifiable. These can be called landmark glomeruli'. Furthermore, other landmark structures, such as the antennal nerve, prominent nerve tracts (e.g. the $\mathrm{T} 1$ tract within the AL of honeybees), cell clusters or axon bundles (like the 'lateral passage' in honeybees) are useful reference structures. Starting from these fixed points, glomeruli can be identified: many of these have characteristic shapes and sizes. However, there are areas of the AL containing glomeruli of similar size and shape. Unless the staining of the glomerular borders is exceptionally good, glomerular identification is difficult in these areas. It is for this reason that some of our identified glomeruli are attributed unambiguously, while others are listed with a question mark, or as a list of several possible candidates. In several instances the glomerular border was so poorly visible in the confocal images that no identification was possible.

\section{Mismatch between electrophysiology and calcium imaging}

When we saw an increase in spike frequency to a certain odour, we generally also found a calcium increase in the imaging. Similarly, an odour response consisting of a spike frequency reduction was correlated with intracellular calcium decrease. Off-inhibitions in the electrophysiological trace were not visible as fast calcium decreases in the imaging (see Fig. 1). The match between spike-trains and calcium concentration changes was true for all PNs recorded, but we had two LNs where this match was not apparent. In these instances, the electrophysiologically recorded neuron and the imaged neuron may be two different cells. For example, if the neuron with the best electrophysiological contact lies 
deep in the AL, in an area which is not accessible with the CCD camera, but superficial neurons are also weakly impaled in a multiple neuron recording, their responses might be clear in the imaging, even though not detectable in the electrophysiology. In three cases (two LNs and one $\mathrm{PN}$ ) the response profile in the imaging was broader than in the electrophysiological recordings. This finding has important implications for how olfactory information is processed in the AL. Most of the $\mathrm{Ca}^{2+}$ increase measured may enter the cell via voltage-sensitive calcium channels, and thus be directly dependent on membrane potential, which in turn determines action potential rate. However, membrane potential changes may be locally modulated, and the driving force at the spike-initiating zone may be locally reduced by inhibitory synapses. This would result in fewer spikes in the neuron output than predicted from the local calcium increase, which is exactly what we found. Furthermore, an important proportion of the excitatory input to both LNs and PNs comes from OSNs. Their transmitter is believed to be acetylcholine (ACh) (Kreissl and Bicker 1989). The honeybee ACh receptor has been shown to be calcium-permeable (Goldberg et al. 1999), accounting for part of the calcium signal measured. This calcium influx may not correlate with spiking output when the neuron is also inhibited by other neurons, again suggesting a more restricted electrophysiological response profile as compared to the calcium imaging. This corresponds to our observations. A broader calcium response in dendritic cell compartments as compared to the electrophysiological output of the cell is also known from olfactory receptor neurons (Zufall et al. 2000). A strong and positive correlation between membrane potential and calcium concentration in simultaneous electrophysiological and calcium recordings has been reported in several systems, including the dendrites of rat mitral cells (Charpak et al. 2001) and visual interneurons of the blowfly (Kurtz et al. 2001; Single and Borst 2002). All these observations together argue that the calcium signal observed is dominated by postsynaptic calcium, and not due to the calcium increase associated with synaptic vesicle release.

\section{Uniglomerular projection neurons}

The calcium responses in the PNs were a good mimic of the electrophysiological responses, even though these measurements were done at different points in time (Figs. 1, 3). This indicates that the postsynaptic intracellular calcium concentration in the PNs is a function of their membrane potential, as has been shown in other systems (Charpak et al. 2001; Kurtz et al. 2001; Single and Borst 2002). The role that this calcium plays in the cell is unknown. While it may be a mere by-product of the calcium permeability of the insect acetylcholine receptors (Goldberg et al. 1999; Single and Borst 2002), it may also have a functional significance, and be involved in short-term memory processes at the level of the
AL (Menzel 2001). Indeed, the calcium response often outlasted the electrophysiological activity, which would support this assertion. Furthermore, the correlated calcium increase in the soma, where input synapses are lacking, suggests that voltage-sensitive calcium channels are expressed on these cells. This supports a functional role of the calcium influx, possibly in relation to the induction of longer-lasting forms of cellular plasticity.

Uniglomerular PNs have their entire and very dense branching pattern within the one innervated glomerulus. The axon then leaves the glomerulus and takes a quite contorted route out of the AL (Fig. 2). Along its way there are two places where morphological structures suggest an interaction with other cells: one close to the glomerulus, and one at the exit of the AL (Fig. 2). The contorted path of the axon may be instrumental in adjusting the travelling time of the APs on their way to the MB and LPL. The short fibres protruding from the axons at the exit of the AL may act as filters if they receive inhibitory input of sufficient efficacy to affect some of the APs (Müller et al. 2002). If that is the case, there may be APs observed when electrophysiologically recording in the AL that do not reach the Kenyon cells in the MB. More research is needed to investigate this question.

In many cases in which we could identify the glomerulus the responses found in PNs matched those reported in the functional atlas (Fig. 8, Table 1). The functional atlas is based on bath-applied calcium-sensitive dyes, a method which emphasizes the response properties of the OSNs innervating the glomeruli (Galizia et al. 1998). Consequently the response profile of a uniglomerular PN is dominated by the responses of the OSNs innervating its glomerulus. Corresponding olfactory response profiles between input and output of an olfactory glomerulus have also been proposed for the Drosophila AL (Ng et al. 2002; Wang et al. 2003). However, other studies have shown that the glomerulus does not just act as a collection of OSNs: results from several species show that the AL 're-formats' the incoming information (Friedrich and Stopfer 2001; Sachse and Galizia 2002). What, then, is the relationship between the incoming information (as known from the functional atlas) and the output (as measured in the PNs)? In two animals we recorded from a PN innervating glomerulus T1-33, which has been well characterized in the functional atlas (Fig. 8). Both responded to octanol and did not respond to citral, as would be predicted from the atlas. However, they differed in other properties, in particular in the degree of spontaneous activity and in the duration of the olfactory response. Thus, different PNs from the same glomerulus may encode different aspects about the stimulus, even when they all respond to the same odour profile. These aspects may include temporal stimulus properties, context information (Christensen et al. 2000; Vickers et al. 2001), as well as memory traces. Differences between fundamental response properties (response latency, timecourse and spectrum) have been shown between 1ACT and mACT PNs in honeybees (Müller et al. 2002). 
Local interneurons: hetero-LNs

We measured a total of 27 hetero-LNs. In all cases, we only found a calcium response in the main glomerulus, and could not detect a response in the glomeruli that were only weakly innervated. Since the calcium measured corresponds to predominantly postsynaptic calcium entry (see above), this finding suggests that the hetero-LNs are polarized: the processes in the main glomerulus receive synaptic input (and may well also form output synapses), while the processes reaching into the weakly innervated glomeruli may only form output synapses. This is further supported by the finding that these neurons' responses generally corresponded to the olfactory response profiles predicted by the functional atlas of the honeybee (Galizia et al. 1999b; Sachse et al. 1999). The functional atlas is based on the afferent OSN input to each glomerulus and these neurons synapse onto LNs. Again, the absence of calcium responses in the weakly innervated glomeruli, even for odours for which we know that these glomeruli get very strong input from the OSNs, supports the proposed polarity of hetero-LNs: input (and possibly also output) in the main glomerulus, output only in the remainder of its branching tree. Hetero-LNs branch diffusely in a limited number of glomeruli. The fact that the OSN input to their main glomerulus dominates their response profile suggests that hetero-LNs are implicated in a glomerulusspecific reshaping of olfactory responses (Sachse and Galizia 2002). Their effect is likely to be inhibitory (Bicker 1999). They probably modulate the slow temporal properties of PN responses, or both slow and fast components. Recent studies have investigated the importance of these slow temporal properties for olfactory coding (Friedrich and Stopfer 2001).

Aspects common to uniglomerular PNs and hetero-LNs

\section{The role of calcium in olfactory neurons}

Calcium rises in the cell bodies matched those in the glomerulus, generally with a time lag, and reduced maximal changes. The temporal resolution $(6 \mathrm{~Hz})$ is not a priori sufficient to exclude that this calcium passively diffused into the soma from the main dendrite. However, when the main neurite reaching to the soma was in focus calcium could be seen to diffuse from the soma into its neurite, showing that the calcium influx originated in the soma membrane (data not shown). Whether it is due to voltage-sensitive calcium channels in the cell body membrane or due to intracellular messengers, and possibly involving intracellular calcium stores, remains to be elucidated. The fact, however, that the cell body responds to odours that evoke a strong calcium signal may be related to learning and memory. Pairing an odour with a sugar-water reward leads to a stable associative olfactory memory in honeybees. Part of the memory trace is localized in the AL (Faber et al. 1999; Menzel 2001). Calcium increases are crucial for this process, as is the increased activity of PKA following the intracellular calcium increase (Müller 2000), and thus $\mathrm{Ca}^{2+}$ and other intracellular signals may converge in the nucleus initiating the transition from short to longlasting forms of plasticity.

All roles for calcium known so far involve intracellular calcium increases. We have shown here that odours can also lead to calcium decreases. Within the main glomerulus this calcium decrease corresponds to a cellmembrane hyperpolarization, which we could observe in the intracellular recordings and in the spike trains. However, whether intracellular calcium decreases in the cell body also have a functional role remains to be determined.

\section{Match of neuron responses with AL atlas}

In many cases the physiological response properties of the cells corresponded to the response profile of the innervated glomerulus as described in the physiological AL atlas (Galizia et al. 1999b; Sachse et al. 1999). However, the number of tested odours in this study, and the overlap with the odours for which the responses are known from the physiological atlas, are still modest. More research is needed to characterize the relationship between the physiological properties of individual neurons and the innervated glomerulus, and to understand the respective role played by different PNs innervating the same glomerulus.

We also found cases of clear mismatch between the physiological atlas and the response recorded in the neuron. In order to understand whether this 'mismatch' is due to intraspecific variability in the glomerular response profiles, or whether individual neurons may not follow the overall glomerular response profile, it will be necessary to combine electrophysiological recordings with calcium imaging of all cells, e.g. by bath-applying a calcium-sensitive dye (Galizia et al. 1999a).

\section{Concluding remarks}

In this study, we analyzed individual PNs or small groups of LNs in the honeybee AL. By combining electrophysiology with calcium imaging, we have access both to fast temporal response properties and the spatial extent of activity. Subsequently, we confocally reconstructed the cells and identified them. Since the innervated glomeruli and surrounding brain landmarks are known, we could relate these cells to the standardized atlas of the honeybee brain. These cells are therefore the first entries in a database of honeybee olfactory cells that will grow with every new cell recorded. Together with the morphological atlas of the AL (Flanagan and Mercer 1989a; Galizia et al. 1999a) and the entire brain (Menzel et al. 2002), and the physiological atlas of the 
honeybee olfactory glomeruli (Galizia et al. 1999b; Sachse et al. 1999), such a database will allow us to use new approaches to investigate the olfactory system. In particular, it will be possible to create computational models of the olfactory code that pool distinct experiments and consider different depths of analysis: single cell, glomerular unit, entire brain. With its relatively limited number of neurons and at the same time remarkable olfactory and cognitive capability (Menzel and Giurfa 2001), the honeybee brain is an ideal study case for such a virtual brain atlas. The honeybee brain database will complement similar efforts taken to understand the mammalian brain (Mazziotta et al. 2001; Toga and Thompson 2001).

Acknowledgments Thanks to Beate Eisermann for help with the figures, Astrid Klawitter for technical assistance, Robert Brandt for help with Amira, Ulrike Schröter for cell tracing, Carsten Duch for help with the electrophysiology, and Mary Wurm for help with the English. Thanks to Randolf Menzel, Silke Sachse and Ulrike Schröter for fruitful comment on the manuscript. The Volkswagenstiftung (I/75-399), the DFG (GRK 120), the BMBF (PTJ0311562X), and HFSP (RGY0050/2001) funded this work.

Animations of the calcium measurements shown in the figures can be accessed under http://galizia.ucr.edu/singleneurons.

\section{References}

Abel R, Rybak J, Menzel R (2001) Structure and response patterns of olfactory interneurons in the honeybee, Apis mellifera. J Comp Neurol 437:363-383

Berg BG, Almaas TJ, Bjaalie JG, Mustaparta H (1998) The macroglomerular complex of the antennal lobe in the tobacco budworm moth Heliothis virescens: specified subdivision in four compartments according to information about biologically significant compounds. J Comp Physiol A 183:669-682

Berg BG, Galizia CG, Brandt R, Mustaparta H (2002) Digital atlases of the antennal lobe in two species of tobacco budworm moths, the oriental Helicoverpa assulta (male) and the American Heliothis virescens (male and female). J Comp Neurol 446:123134

Bicker G (1999) Histochemistry of classical neurotransmitters in antennal lobes and mushroom bodies of the honeybee. Microsc Res Tech 45:174-183

Boeckh J, Tolbert LP (1993) Synaptic organization and development of the antennal lobe in insects. Microsc Res Tech 24:260 280

Bornhauser BC, Meyer EP (1997) Histamine-like immunoreactivity in the visual system and brain of an orthopteran and a hymenopteran insect. Cell Tissue Res 287:211-221

Bruyne M de, Clyne PJ, Carlson JR (1999) Odor coding in a model olfactory organ: the Drosophila maxillary palp. J Neurosci 19:4520-4532

Bruyne M de, Foster K, Carlson JR( 2001) Odor coding in the Drosophila antenna. Neuron 30:537-552

Charpak S, Mertz J, Beaurepaire E, Moreaux L, Delaney K (2001) Odor-evoked calcium signals in dendrites of rat mitral cells. Proc Natl Acad Sci USA 98:1230-1234

Christensen TA, Waldrop BR, Harrow ID, Hildebrand JG (1993) Local interneurons and information processing in the olfactory glomeruli of the moth Manduca sexta. J Comp Physiol A 173:385-399

Christensen TA, Pawlowski VM, Lei H, Hildebrand JG (2000) Multi-unit recordings reveal context-dependent modulation of synchrony in odor-specific neural ensembles. Nat Neurosci 3:927-931
Distler PG, Boeckh J (1998) An improved model of the synaptic organization of insect olfactory glomeruli. Ann N Y Acad Sci 855:508-510

Faber T, Joerges J, Menzel R (1999) Associative learning modifies neural representations of odors in the insect brain. Nat Neurosci 2:74-78

Flanagan D, Mercer AR (1989a) An atlas and 3-D reconstruction of the antennal lobes in the worker honey bee, Apis mellifera $\mathrm{L}$. (Hymenoptera: Apidae). Int J Insect Morphol Embryol 18:145159

Flanagan D, Mercer AR (1989b) Morphology and response characteristics of neurones in the deutocerebrum of the brain in the honeybee Apis mellifera. J Comp Physiol A 164:483494

Fonta C, Sun XJ, Masson C (1993) Morphology and spatial distribution of bee antennal lobe interneurones responsive to odours. Chem Senses 18:101-119

Friedrich RW, Laurent G (2001) Dynamic optimization of odor representations by slow temporal patterning of mitral cell activity. Science 291:889-894

Friedrich RW, Stopfer M (2001) Recent dynamics in olfactory population coding. Curr Opin Neurobiol 11:468-474

Galizia CG, Menzel R (2001) The role of glomeruli in the neural representation of odours: results from optical recording studies. J Insect Physiol 47:115-130

Galizia CG, Joerges J, Kuttner A, Faber T, Menzel R (1997) A semi-in vivo preparation for optical recording of the insect brain. J Neurosci Methods 76:61-69

Galizia CG, Nagler K, Holldobler B, Menzel R (1998) Odour coding is bilaterally symmetrical in the antennal lobes of honeybees (Apis mellifera). Eur J Neurosci 10:2964-2974

Galizia CG, McIlwrath SL, Menzel R (1999a) A digital threedimensional atlas of the honeybee antennal lobe based on optical sections acquired by confocal microscopy. Cell Tissue Res 295:383-394

Galizia CG, Sachse S, Rappert A, Menzel R (1999b) The glomerular code for odor representation is species specific in the honeybee Apis mellifera. Nat Neurosci 2:473-478

Gascuel J, Masson C (1991) A quantitative ultrastructural study of the honeybee antennal lobe. Tissue Cell 23:341-355

Goldberg F, Grunewald B, Rosenboom H, Menzel R (1999) Nicotinic acetylcholine currents of cultured Kenyon cells from the mushroom bodies of the honey bee Apis mellifera. J Physiol (Lond) 514:759-768

Hansson BS, Anton S (2000) Function and morphology of the antennal lobe: new developments. Annu Rev Entomol 45:203231

Hansson BS, Christensen TA (1999) Functional characteristics of the antennal lobe. In: Hansson BS (ed) Insect olfaction. Springer, Berlin Heidelberg New York, pp 125-161

Hansson BS, Ljungberg H, Hallberg E, Lofstedt C (1992) Functional specialization of olfactory glomeruli in a moth. Science 256:1313-1315

Hildebrand JG, Shepherd GM (1997) Mechanisms of olfactory discrimination: converging evidence for common principles across phyla. Annu Rev Neurosci 20:595-631

Homberg U (1984) Processing of antennal information in extrinsic mushroom body neurons of the bee brain. J Comp Physiol A 154:825-836

Kanzaki R, Arbas EA, Strausfeld NJ, Hildebrand JG (1989) Physiology and morphology of projection neurons in the antennal lobe of the male moth Manduca sexta. J Comp Physiol A $165: 427-453$

King JR, Christensen TA, Hildebrand JG (2000) Response characteristics of an identified, sexually dimorphic olfactory glomerulus. J Neurosci 20:2391-2399

Kreissl S, Bicker G (1989) Histochemistry of acetylcholinesterase and immunocytochemistry of an acetylcholine receptor-like antigen in the brain of the honeybee. J Comp Neurol 286:71-84

Kurtz R, Warzecha AK, Egelhaaf M (2001) Transfer of visual motion information via graded synapses operates linearly in the natural activity range. J Neurosci 21:6957-6966 
Laissue PP, Reiter C, Hiesinger PR, Halter S, Fischbach KF, Stocker RF (1999) Three-dimensional reconstruction of the antennal lobe in Drosophila melanogaster. J Comp Neurol 405:543-552

Laurent G (1999) A systems perspective on early olfactory coding. Science 286:723-728

Laurent G, Wehr M, Davidowitz H (1996) Temporal representations of odors in an olfactory network. J Neurosci 16:38373847

Laurent G, Stopfer M, Friedrich RW, Rabinovich MI, Volkovskii A, Abarbanel HD (2001) Odor encoding as an active, dynamical process: experiments, computation, and theory. Annu Rev Neurosci 24:263-297

MacLeod K, Laurent G (1996) Distinct mechanisms for synchronization and temporal patterning of odor-encoding neural assemblies. Science 274:976-979

Malun D (1991) Inventory and distribution of synapses of identified uniglomerular projection neurons in the antennal lobe of Periplaneta americana. J Comp Neurol 305:348-360

Mazziotta J, Toga A, Evans A, Fox P, Lancaster J, Zilles K, Woods R, Paus T, Simpson G, Pike B, Holmes C, Collins L, Thompson P, MacDonald D, Iacoboni M, Schormann T, Amunts K, Palomero-Gallagher N, Geyer S, Parsons L, Narr K, Kabani N, Le Goualher G, Boomsma D, Cannon T, Kawashima R, Mazoyer B (2001) A probabilistic atlas and reference system for the human brain: international consortium for brain mapping (ICBM). Philos Trans R Soc Lond B 356:12931322

Menzel R (2001) Searching for the memory trace in a mini-brain, the honeybee. Learn Mem 8:53-62

Menzel R, Giurfa M (2001) Cognitive architecture of a mini-brain: the honeybee. Trends Cogn Sci 5:62-71

Menzel R, Brandt R, Rohlfing T, Zoeckler M, Stollhoff N (2002) The virtual bee brain atlas - a novel way to document neuroanatomical data. In: XIV International Congress of IUSSI. IUSSI, Sapporo, Japan, p 28

Müller D, Abel R, Brandt R, Zockler M, Menzel R (2002) Differential parallel processing of olfactory information in the honeybee, Apis mellifera L. J Comp Physiol A 188:359370

Müller U (2000) Prolonged activation of cAMP-dependent protein kinase during conditioning induces long-term memory in honeybees. Neuron 27:159-168

Ng M, Roorda RD, Lima SQ, Zemelman BV, Morcillo P, Miesenbock G (2002) Transmission of olfactory information between three populations of neurons in the antennal lobe of the fly. Neuron 36:463-474

Rospars JP (1988) Structure and development of the insect antennodeutocerebral system. Int J Insect Morphol Embryol 17:243-294

Rospars JP, Chambille I (1981) Deutocerebrum of the cockroach Blaberus craniifer Burm. Quantitative study and automated identification of the glomeruli. J Neurobiol 12:221-247

Røstelien T, Borg-Karlson AK, Fäldt J, Jacobsen U, Mustaparta H (2000) The plant sesquiterpene germacrene D specifically activates a major type of antennal receptor neruons of the tobacco budworm moth Heliothis virescens. Chem Senses 25 : $141-148$

Sachse S, Galizia CG (2002) Role of inhibition for temporal and spatial odor representation in olfactory output neurons: a calcium imaging study. J Neurophysiol 87:1106-1117

Sachse S, Rappert A, Galizia CG (1999) The spatial representation of chemical structures in the antennal lobe of honeybees: steps towards the olfactory code. Eur J Neurosci 11:3970-3982

Sadek MM, Hansson BS, Rospars JP, Anton S (2002) Glomerular representation of plant volatiles and sex pheromone components in the antennal lobe of the female Spodoptera littoralis. J Exp Biol 205:1363-1376

Schäfer S, Bicker G (1986) Distribution of GABA-like immunoreactivity in the brain of the honeybee. J Comp Neurol 246:287300

Single S, Borst A (2002) Different mechanisms of calcium entry within different dendritic compartments. J Neurophysiol 87:1616-1624

Stocker RF, Lienhard MC, Borst A, Fischbach KF (1990) Neuronal architecture of the antennal lobe in Drosophila melanogaster. Cell Tissue Res 262:9-34

Stopfer M, Bhagavan S, Smith BH, Laurent G (1997) Impaired odour discrimination on desynchronization of odour-encoding neural assemblies. Nature 390:70-74

Stranden M, Borg-Karlson A-K, Mustaparta H (2002) Receptor neuron discrimination of germacrene $\mathrm{D}$ enantiomers. Chem Senses 27:143-152

Sun X-J, Fonta C, Masson C (1993) Odour quality processing by bee antennal lobe interneurones. Chem Senses 18:355-377

Toga AW, Thompson PM (2001) Maps of the brain. Anat Rec 265:37-53

Vickers NJ, Christensen TA, Hildebrand JG (1998) Combinatorial odor discrimination in the brain: attractive and antagonist odor blends are represented in distinct combinations of uniquely identifiable glomeruli. J Comp Neurol 400:35-56

Vickers NJ, Christensen TA, Baker TC, Hildebrand JG (2001) Odour-plume dynamics influence the brain's olfactory code. Nature 410:466-470

Vosshall LB, Wong AM, Axel R (2000) An olfactory sensory map in the fly brain. Cell 102:147-159

Wang JW, Wong AM, Flores J, Vosshall LB, Axel R (2003) Twophoton calcium imaging reveals an odor-evoked map of activity in the fly brain. Cell 112:271-282

Wehr M, Laurent G (1996) Odour encoding by temporal sequences of firing in oscillating neural assemblies. Nature 384:162-166

Witthöft W (1967) Absolute Anzahl und Verteilung der Zellen im Hirn der Honigbiene. Z Morphol Tiere 61:160-184

$\mathrm{Xu} \mathrm{F}$, Greer CA, Shepherd GM (2000) Odor maps in the olfactory bulb. J Comp Neurol 422:489-495

Zufall F, Leinders-Zufall T, Greer CA (2000) Amplification of odor-induced $\mathrm{Ca}(2+)$ transients by store-operated $\mathrm{Ca}(2+)$ release and its role in olfactory signal transduction. J Neurophysiol 83:501-512 